\title{
A multi-phase transition model for dislocations with interfacial microstructure
}

\author{
SIMONE CACACE \\ CERMICS - ENPC, 6 et 8 avenue Blaise Pascal, Cité Descartes - Champs sur Marne, \\ 77-455 Marne la Vallée Cedex 2, France \\ E-mail: cacaes@cermics.enpc.fr \\ ADRIANA GARRONI \\ Dipartimento di Matematica “G. Castelnuovo”, Università di Roma "La Sapienza”, \\ Piazzale A. Moro, 2, 00185 Roma, Italy \\ E-mail: garroni@mat.uniromal.it
}

[Received 12 January 2008]

\begin{abstract}
We study, by means of $\Gamma$-convergence, the asymptotic behavior of a variational model for dislocations moving on a slip plane. The variational functional is a two-dimensional multi-phase transition-type energy given by a nonlocal term and a nonlinear potential which penalizes noninteger values for the components of the phase. In the limit we obtain an anisotropic sharp interfaces model. The relevant feature of this problem is that optimal sequences in general are not given by a onedimensional profile, but they can create microstructure.
\end{abstract}

\section{Introduction}

We study, by means of $\Gamma$-convergence, the asymptotic behavior as $\varepsilon \rightarrow 0$ of the functionals

$$
\begin{aligned}
F_{\varepsilon}(u)= & \frac{1}{|\log \varepsilon|} \int_{T^{2}} \int_{T^{2}}(u(x)-u(y))^{T} \mathbb{J}(x-y)(u(x)-u(y)) \mathrm{d} x \mathrm{~d} y \\
& +\frac{1}{\varepsilon|\log \varepsilon|} \int_{T^{2}} \operatorname{dist}^{2}\left(u(x), \mathbb{Z}^{N}\right) \mathrm{d} x,
\end{aligned}
$$

where $\varepsilon>0$ is a small parameter, $T^{2}=\mathbb{R}^{2} / \mathbb{Z}^{2}$ is the unit torus of $\mathbb{R}^{2}, u: T^{2} \rightarrow \mathbb{R}^{N}$ is a 1-periodic vector field, $\mathbb{J}: T^{2} \rightarrow \mathcal{M}^{N \times N}$ is a singular matrix-valued kernel which defines a quadratic form equivalent to the square of the $H^{1 / 2}$ seminorm of $u$ and $\operatorname{dist}\left(\cdot, \mathbb{Z}^{N}\right)$ is the distance function from the set $\mathbb{Z}^{N}$ of multi-integers.

An energy of type (1) has been proposed by Koslowski and Ortiz ([17], and also [16]) as a multi-phase model for planar dislocations in crystals, inspired by the classical model of NabarroPeierls (see [8]). When an external stress is imposed, the specific geometry of the crystal constrains deformations along planes (slip planes), on which some preferred slip directions (slip systems) are identified. This process, known as crystallographic slip, may produce crystal lattice defects of topological kind. Dislocations are one-dimensional defects that can move along slip planes with a low energy cost, drastically modifying the mechanical properties of the crystal. In particular, dislocations are responsible for many interesting phenomena, such as plasticity and hardening ([8]). In [17] the authors consider an elastic crystal with a single slip plane undergoing periodic plastic slips, described by a vector field $u$ (defined on the torus $T^{2}$ ). The plastic slip is given by a linear 
combination of $N$ slip systems (with coefficients given by $u$ ), where $N$ depends on the geometry of the crystal. In the functional (1), the first term represents the long-range elastic distortion induced by the slip and the second term penalizes slips that are not compatible with the lattice structure.

The asymptotic behavior of the energy (1) as $\varepsilon \rightarrow 0$ provides an anisotropic line-tension model for line defects, in which dislocations are identified with the discontinuity lines of a $\mathbb{Z}^{N}$-valued slip field where all the energy concentrates. It is well known that $\Gamma$-convergence of energies is essentially equivalent to the convergence of the corresponding minimum problems and that it is stable with respect to the addition of lower-order terms (like external stresses or a twist boundary condition). This means that a minimum problem involving the Nabarro-Peierls model can be replaced by the corresponding minimum problem for the line-tension model.

In analytical terms, the functional (1) belongs to the class of multi-well functionals singularly perturbed by a higher-order term. We show that their limit is given by a sharp interface model as expected in these cases. Nevertheless, the interest of these functionals lies in that the behavior of the optimal sequences may be very different from the usual one and the interfaces may produce microstructure, which is the main result of this paper.

Starting with the well-known $\Gamma$-convergence result by Modica and Mortola ([20]), related to the Cahn-Hilliard model for two-phase fluids (see [12] and [19]), a large literature is now available where sharp interface models describe the asymptotic behavior of multi-well potentials singularly perturbed by local regularizations of Dirichlet type (see e.g. [9] and [10]) or nonlocal terms described by integrable interaction kernels, as in the Ising models with Kac potentials (see for instance [1] $-[3]$ ). In all these cases it is well known that the line tension energy density of the $\Gamma$-limit is characterized by an optimal-profile problem, involving both terms of the approximating energy, the nonconvex potential and the singular perturbation.

More recently, the analysis of nonlocal variants of the Cahn-Hilliard model, namely phase transitions with a boundary line tension, led to the study of functionals in which the perturbation corresponds to the $H^{1 / 2}$ seminorm, i.e., it is described by a nonintegrable interaction kernel with critical singularity $([4],[5])$. Due to the singularity of the interaction kernel a logarithmic rescaling of the functional is needed in order to produce a nontrivial limit energy; the effect of this rescaling is that the $\Gamma$-limit functional does not require an optimal-profile problem, all transitions between two wells are optimal as far as they occur on a layer of width $\varepsilon$ around the interface. Moreover, the line tension energy density does not depend on the precise shape of the potential, but only on the position of its zeros.

The functional of dislocations (1) is a generalization of the case considered in [4]: the nonlocal term is anisotropic, i.e., it is only equivalent to the $H^{1 / 2}$ seminorm, and the set of wells of the potential in the local term is not compact (see also [18]).

A first $\Gamma$-convergence result for functionals (1) has been obtained in [15] under the additional assumption that the crystallographic slip is driven by a single slip direction. This condition modifies the nature of the functional reducing it to a functional of the same type, but defined on scalar functions. In this case the $\Gamma$-limit functional is an anisotropic sharp interface functional of the form

$$
\int_{S_{u}} \gamma(n)|[u]| \mathrm{d} \mathcal{H}^{1}
$$

where $u$ is an integer-valued phase-field representing the slip in a given direction, $S_{u}$ is the jump set of $u, n_{u}$ the normal vector of $S_{u}$ and $[u]$ the jump of $u$. The behavior of optimal sequences in this case is similar to what happens in the simpler case studied in [4]. The transition between two phases 
does not depend on the specific choice of the nonconvex potential that penalizes the misfit of the lattice and occurs along flat optimal interfaces. In particular, optimal sequences can be obtained by mollifying the limit configuration at a scale $\varepsilon$. This implies that the anisotropic line tension energy density $\gamma(n)$ is explicitly computed by integrating the anisotropic kernel on fibers orthogonal to the vector $n$.

Dealing with the general case, we find out a relevant difference with respect to the scalar case. We can in fact show that optimal sequences in general are not given by one-dimensional interfaces and can occur in complex patterns, strongly depending on the normal to the singular set of the slip fields, which takes into account the anisotropy of the elastic interactions between the atoms of the crystal. This makes the identification of the $\Gamma$-limit of functionals (1) a hard task and suggests approaching the problem in a nonconstructive way. By means of integral representation theory for $\Gamma$-limits defined on Caccioppoli partitions ([6], [7]) we prove (see Section 3p that there exists a subsequence $\varepsilon_{h} \rightarrow 0$ and a function $\varphi: \mathbb{Z}^{N} \times S^{1} \rightarrow \mathbb{R}$ such that the functionals $F_{\varepsilon_{h}} \Gamma$-converge to

$$
\int_{S_{u}} \varphi\left([u], n_{u}\right) \mathrm{d} \mathcal{H}^{1}, \quad u \in B V\left(T^{2}, \mathbb{Z}^{N}\right) .
$$

In Section 4 by means of an explicit example in the simple case of a cubic lattice, we show that the flat interfaces are in general not optimal. Indeed, we construct a sequence of transitions that produce interfacial microstructure and that give an energy lower than the one obtained with one-dimensional transitions.

The main point of this phenomenon is that the limit energy obtained by taking only flat interfaces (i.e. by mollification of the limit configurations) is in general not lower semicontinuous and its relaxation is responsible for the formation of microstructure. The question that remains open is whether this relaxation is actually the $\Gamma$-limit that we construct abstractly. We conjecture that this is the case.

\section{The functional of dislocations}

\subsection{Derivation of the multi-phase model}

In this section we briefly discuss the phase-transition model for dislocations proposed by Koslowski and Ortiz in [17] (see also [16]). For the detailed rescaling argument in the derivation of the model see [14]. We consider an elastic crystal, in the context of small strains, on which the plastic slip occurs only on one plane, the plane $x_{3}=0$. Following the Nabarro-Peierls model for dislocations, in absence of an applied force-field, the total energy of the crystal is then given by

$$
E=E^{\text {Elastic }}+E^{\text {Peierls }} .
$$

The first term $E^{\text {Elastic }}$ is the long range elastic interaction energy and represents the elastic distortion

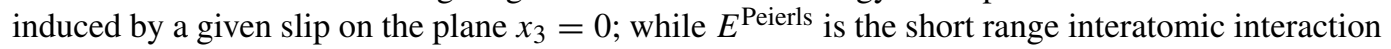
energy and can be expressed by an interplanar potential which describes the misfits of the crystal lattice due to the slip.

We assume the additional condition that the slip is periodic and so, after rescaling, we identify the slip plane with the torus $T^{2}$ of $\mathbb{R}^{2}$. The plastic slip $v: T^{2} \rightarrow \mathbb{R}^{2}$ is then identified with a vector field $u: T^{2} \rightarrow \mathbb{R}^{N}$ that describes the plastic deformations induced by $N$ slip systems, where $N$ depends on the crystal lattice structure. More precisely, if $\left\{\mathbf{s}_{i}\right\}_{i=1, \ldots, N}$ are the $N$ slip directions 
active on the slip plane $T^{2}$, the slip field $v$ can be expressed through the multi-phase slip field $u: T^{2} \rightarrow \mathbb{R}^{N}$ as follows:

$$
v=\sum_{i=1}^{N} u_{i} \mathbf{s}_{i}
$$

where $u_{i}$ is the $i$ th component of $u$. Then the misfit energy of the crystal attains its minima when all the components of $u$ are integral multiples of slip directions, i.e.,

$$
u_{i}=\xi_{i} \mathbf{s}_{i}, \quad \xi_{i} \in \mathbb{Z}, \quad i=1, \ldots, N .
$$

These special slips determine the location of the wells of the interplanar potential $W$, which can be given for instance by the following piecewise quadratic function:

$$
W(u)=\frac{\mu}{2 \varepsilon} \sum_{i=1}^{N} \min _{\xi_{i} \in \mathbb{Z}}\left|u_{i}-\xi_{i}\right|^{2}=\frac{\mu}{2 \varepsilon} \operatorname{dist}^{2}\left(u, \mathbb{Z}^{N}\right),
$$

where $\varepsilon$ is a small parameter proportional to the interplanar distance and $\mu$ is the elastic modulus of the crystal. Then the short range interaction energy of dislocations is

$$
E^{\text {Peierls }}(u)=\int_{T^{2}} W(u(x)) \mathrm{d} x .
$$

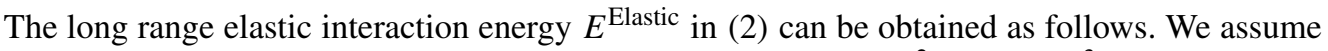
that crystal deformations are described by a displacement field $U: T^{2} \times \mathbb{R} \rightarrow \mathbb{R}^{3}$ and decompose the gradient $\nabla U$ in the Kröner additive form:

$$
\nabla U=\beta^{e}+\beta^{p}
$$

where $\beta^{e}, \beta^{p}$ denote the elastic and plastic distortion tensors respectively. Since crystallographic slip is all supported on $T^{2}$ we write the plastic distortion as

$$
\beta^{p}=\left([U] \otimes e_{3}\right) \mathcal{H}^{2}\left\llcorner T^{2},\right.
$$

where $[U]=\left(\left[U_{1}\right],\left[U_{2}\right],\left[U_{3}\right]\right)$ is the jump of the displacement across the slip plane, $e_{3}=$ $(0,0,1)$ is the unit normal to $T^{2}$, and $\mathcal{H}^{2}\left\llcorner T^{2}\right.$ represents the two-dimensional Hausdorff measure concentrated on $T^{2}$. It follows that

$$
\beta^{p}\left(x_{1}, x_{2}, x_{3}\right)=\left(\begin{array}{ccc}
0 & 0 & {\left[U_{1}\right]\left(x_{1}, x_{2}\right)} \\
0 & 0 & {\left[U_{2}\right]\left(x_{1}, x_{2}\right)} \\
0 & 0 & 0
\end{array}\right) \mathcal{H}^{2}\left\llcorner T^{2}\right.
$$

Then we can obtain the long range elastic interactions energy $E^{\text {Elastic }}$, induced by the slip $v=$ $\sum_{i=1}^{N} u_{i} \mathbf{s}_{i}$, by solving the following minimum problem:

$$
E^{\text {Elastic }}(v)=\min _{[U]=v} \mathcal{E}(U),
$$

where $\mathcal{E}(U)$ is the linear isotropic elastic energy

$$
\mathcal{E}(U)=\int_{T^{2} \times \mathbb{R}}\left(\mu\left|e\left(\beta^{e}\right)\right|^{2}+\frac{\lambda}{2}\left|\operatorname{tr} e\left(\beta^{e}\right)\right|^{2}\right) \mathrm{d} x_{1} \mathrm{~d} x_{2} \mathrm{~d} x_{3},
$$

$e\left(\beta^{e}\right)=\frac{1}{2}\left(\beta^{e}+\beta^{e T}\right)$ and $\mu, \lambda$ are the Lamé coefficients of the crystal. 
The minimization in (7) can be explicitly carried out by means of Fourier variables. It follows that

$$
E^{\text {Elastic }}(\widehat{v})=\frac{1}{(2 \pi)^{2}} \frac{\mu}{4} \sum_{k \in 2 \pi \mathbb{Z}^{2}} \widehat{v}(k)^{T} \widehat{\mathcal{A}}(k) \widehat{v}^{*}(k),
$$

where the interaction matrix $\widehat{\mathcal{A}}$ is given by

$$
\widehat{\mathcal{A}}(k)=\left(\begin{array}{cc}
\frac{1}{1-v} \frac{k_{1}^{2}}{|k|}+\frac{k_{2}^{2}}{|k|} & \frac{v}{1-v} \frac{k_{1} k_{2}}{|k|} \\
\frac{v}{1-v} \frac{k_{1} k_{2}}{|k|} & \frac{1}{1-v} \frac{k_{2}^{2}}{|k|}+\frac{k_{1}^{2}}{|k|}
\end{array}\right)
$$

and $v=\frac{\lambda}{2(\mu+\lambda)}$ is the Poisson ratio of the crystal. It is easy to check that $\widehat{\mathcal{A}}$ has the two eigenvalues

$$
\lambda_{1}=|k|, \quad \lambda_{2}=\frac{1}{1-v}|k|, \quad v \in(-1,1 / 2) .
$$

Then 9 defines a positive quadratic form equivalent to the square of the $H^{1 / 2}$ seminorm. More precisely,

$$
C_{1}[v]_{H^{1 / 2}\left(T^{2}\right)}^{2} \leqslant E^{\text {Elastic }}(\widehat{v}) \leqslant C_{2}[v]_{H^{1 / 2}\left(T^{2}\right)}^{2},
$$

where

$$
C_{1}=\frac{1}{(2 \pi)^{2}} \frac{\mu}{4}, \quad C_{2}=\frac{1}{1-v} \frac{1}{(2 \pi)^{2}} \frac{\mu}{4}
$$

and

$$
[v]_{H^{1 / 2}\left(T^{2}\right)}=\left(\sum_{k \in 2 \pi \mathbb{Z}^{2}}|k||\widehat{v}(k)|^{2}\right)^{1 / 2} .
$$

In space variables and in terms of the multi-phase $u$, the energy (9) can be rewritten as

$$
E^{\text {Elastic }}(u)=\frac{\mu}{2} \int_{T^{2}} \int_{T^{2}}(u(x)-u(y))^{T} \mathbb{J}(x-y)(u(x)-u(y)) \mathrm{d} x \mathrm{~d} y,
$$

where $\mathbb{J}: T^{2} \rightarrow \mathcal{M}^{N \times N}$ is given by

$$
\mathbb{J}_{i j}(x):=\sum_{k \in \mathbb{Z}^{2}} \mathbf{s}_{i}^{T} \mathbb{J}_{0}(x+k) \mathbf{s}_{j}, \quad i, j=1, \ldots, N,
$$

and $\mathbb{J}_{0}$ is the following matrix homogeneous of degree -3 :

$$
\mathbb{J}_{0}(x)=\frac{1}{8 \pi(1-v)|x|^{3}}\left(\begin{array}{cc}
v+1-3 v \frac{x_{2}^{2}}{|x|^{2}} & 3 v \frac{x_{1} x_{2}}{|x|^{2}} \\
3 v \frac{x_{1} x_{2}}{|x|^{2}} & v+1-3 v \frac{x_{1}^{2}}{|x|^{2}}
\end{array}\right) .
$$

The following properties hold:

(i) $\mathbb{J}$ is 1-periodic, i.e., defined on $T^{2}$;

(ii) $\mathbb{J}(x)=\mathcal{O}\left(|x|^{-3}\right)$ as $|x| \rightarrow 0$; 
(iii) $\mathbb{I}$ defines a positive quadratic form equivalent to the square of the $H^{1 / 2}$ seminorm;

(iv) $\mathbb{J}$ satisfies

$$
\lim _{\delta \rightarrow 0} \delta^{3}(\mathbb{J}(\delta x))_{i j}=\mathbf{s}_{i}^{T} \mathbb{J}_{0}(x) \mathbf{s}_{j}, \quad i, j=1, \ldots, N,
$$

uniformly on $\left\{x \in \mathbb{R}^{2}:|x| \leqslant \sigma\right\}$ for every $\sigma>0$.

Finally, we put (3) and (13) in (2), we divide by the constant factor $\mu / 2$, and we get

$$
\begin{aligned}
E_{\varepsilon}(u):= & \int_{T^{2}} \int_{T^{2}}(u(x)-u(y))^{T} \mathrm{~J}(x-y)(u(x)-u(y)) \mathrm{d} x \mathrm{~d} y \\
& +\frac{1}{\varepsilon} \int_{T^{2}} \operatorname{dist}^{2}\left(u(x), \mathbb{Z}^{N}\right) \mathrm{d} x .
\end{aligned}
$$

The functional above belongs to the class of multi-well potentials singularly perturbed by a higher-order term. The minimization of this energy with the addition of lower-order terms (as external loads or imposing a twist boundary condition) gives rise to a competition between the two terms in $E_{\varepsilon}$ : the potential forces the slip field $u$ to be close to the phases, namely to assume integer values almost everywhere; while the kernel $\mathbb{J}$ prefers to reduce the long range elastic interactions (the $H^{1 / 2}$ seminorm of $u$ ) by separating the phases as much as possible. This competition is then resolved by slip fields which make transitions between the phases, inside small regions of width proportional to the interatomic distance $\varepsilon$, the so called cores of dislocations. The corresponding elastic distortion due to the presence of the dislocations is of the order of the logarithm of the core radius, i.e., in this transition between two integer multi-phases on a layer of width $\varepsilon$ the field $u$ has an $H^{1 / 2}$ norm of order $|\log \varepsilon|$.

After normalizing the energy by $|\log \varepsilon|$ one expects, in the limit as $\varepsilon$ goes to zero, that the cores of dislocations collapse to lines on which the energy concentrates. Dislocation lines are then described by the singular sets of slip fields of pure phases (i.e., $\mathbb{Z}^{N}$-valued) and the amount of energy carried by each dislocation is proportional to its length, locally weighted by the kernel $\mathbb{J}$ which takes into account the anisotropy of the elastic interactions.

\subsection{What is known in the scalar case}

A first analysis of the asymptotic behavior for the model presented above is due to Garroni and Müller [15] under the special assumption that the crystallographic slip on the slip plane $T^{2}$ is driven by a single slip direction. This assumption reduces the functional to be defined only on scalar slip functions. More precisely, they consider slip fields of the form $u e_{1}$, where $e_{1}$ is the first element of the canonical basis of $\mathbb{R}^{2}$ and $u: T^{2} \rightarrow \mathbb{R}$ is a 1-periodic scalar function. In this case the functional $E_{\varepsilon}$ of dislocations reduces to

$$
E_{\varepsilon}(u):=\int_{T^{2}} \int_{T^{2}} \mathrm{~J}(x-y)|u(x)-u(y)|^{2} \mathrm{~d} x \mathrm{~d} y+\frac{1}{\varepsilon} \int_{T^{2}} \operatorname{dist}^{2}(u(x), \mathbb{Z}) \mathrm{d} x,
$$

where the kernel $\mathrm{J}: T^{2} \rightarrow \mathbb{R}$ is given by

$$
\mathrm{J}(x)=\sum_{k \in \mathbb{Z}^{2}} \mathrm{~J}_{0}(x+k), \quad \mathrm{J}_{0}(x)=\frac{1}{8 \pi(1-v)|x|^{3}}\left(v+1-3 v \frac{x_{2}^{2}}{|x|^{2}}\right)
$$

and has the same properties of the kernel $\mathbb{J}$ stated above.

The following $\Gamma$-convergence result holds. 
THEOREM 1 ([15]) Let $E_{\varepsilon}$ be the energy defined in (14). Then the functional

$$
I_{\varepsilon}(u)= \begin{cases}\frac{1}{|\log \varepsilon|} E_{\varepsilon}(u) & \text { if } u \in H^{1 / 2}\left(T^{2}\right), \\ \infty & \text { otherwise in } L^{1}\left(T^{2}\right),\end{cases}
$$

$\Gamma\left(L^{1}\right)$-converges to

$$
I(u)= \begin{cases}\int_{S_{u}} \gamma(n)|[u]| \mathrm{d} \mathcal{H}^{1} & \text { if } u \in B V\left(T^{2}, \mathbb{Z}\right), \\ \infty & \text { otherwise in } L^{1}\left(T^{2}\right),\end{cases}
$$

where $n \in S^{1}$ is the normal on $S_{u}$, [u] is the jump of $u$ across $S_{u}$ in the direction $n$ and $\gamma(n)$ is an anisotropic line tension which only depends on the kernel J:

$$
\gamma(n):=2 \lim _{\delta \rightarrow 0} \delta^{2} \int_{x \cdot n=\delta} \mathrm{J}(x) \mathrm{d} \mathcal{H}^{1}=2 \int_{x \cdot n=1} \mathrm{~J}_{0}(x) \mathrm{d} \mathcal{H}^{1} .
$$

Moreover, for every sequence $\left\{u_{\varepsilon}\right\} \subseteq L^{1}\left(T^{2}\right)$ such that

$$
\sup _{\varepsilon>0} I_{\varepsilon}\left(u_{\varepsilon}\right)<\infty
$$

there exists a sequence $\left\{a_{\varepsilon}\right\} \subset \mathbb{Z}$ such that $\left\{u_{\varepsilon}-a_{\varepsilon}\right\}$ is bounded in $L^{2}\left(T^{2}\right)$ and strongly pre-compact in $L^{1}\left(T^{2}\right)$; every cluster point of $\left\{u_{\varepsilon}-a_{\varepsilon}\right\}$ belongs to $B V\left(T^{2}, \mathbb{Z}\right)$.

We remark that the density $\gamma(n)$ does not depend on the kernel J, but only on the homogeneous part $\mathrm{J}_{0}$. This implies that the problem is actually not affected by periodic boundary conditions and Theorem 1 still holds for any kernel equivalent to the $H^{1 / 2}$ seminorm.

Due to the lack of coerciveness of the interplanar potentials in $I_{\varepsilon}$, the compactness property stated in Theorem 1 turns out to be a quite subtle result and requires a delicate a priori estimate for the $L^{2}$ norm of sequences $u_{\varepsilon}$ with equibounded energy. On the other hand, the phenomenology of the $\Gamma$-convergence result is similar to what one has for the simpler case considered in [4]. In fact, in [15] it is shown that the optimal sequences for a given function $u \in B V\left(T^{2}, \mathbb{Z}\right)$, with $|[u]|=1$, are given by any sequence of the form $v_{\varepsilon}=u * \varphi_{\varepsilon}$, where $\varphi_{\varepsilon}(x)=\varepsilon^{-2} \varphi(x / \varepsilon)$ is any arbitrary mollifier. We refer to this fact as the flat optimal interface (or one-dimensional optimal profile). As for the case of a limiting configuration with jumps larger than 1 , one has to first split the jumps into jumps of size 1 and then mollify.

The main step in proving the existence of a flat optimal interface is based on a truncation and then a blow-up argument that shows that sequences $u_{\varepsilon}$ with finite energy are essentially one-dimensional interfaces (i.e. $u_{\varepsilon}$ take values almost at the bottom of the wells of the potential up to a small strip of approximate width $\varepsilon$ around the jumps of the limit). As we will see with an explicit example in Section 4 this argument cannot be used in the general case of multiple slip systems (corresponding to the vectorial functional of dislocations). The example shows actually that in the general case we cannot expect a flat optimal interface, i.e., the optimal sequences cannot be constructed explicitly as in the scalar case, since a relaxation phenomenon can produce microstructure for the optimal sequences.

Our strategy to attack the general vector case will then be to prove the existence of the $\Gamma$-limit of the rescaled functional of dislocations in a nonconstructive way, by means of a compactness argument together with an integral representation result. This will be done in the next section. 


\section{The $\Gamma$-limit of the functional of dislocations}

This section is devoted to the $\Gamma$-convergence result as $\varepsilon \rightarrow 0$ for the functional of dislocations, which we rewrite here by introducing logarithmic rescaling:

$$
\begin{aligned}
F_{\varepsilon}(u)= & \frac{1}{|\log \varepsilon|} \int_{T^{2}} \int_{T^{2}}(u(x)-u(y))^{T} \mathbb{J}(x-y)(u(x)-u(y)) \mathrm{d} x \mathrm{~d} y \\
& +\frac{1}{\varepsilon|\log \varepsilon|} \int_{T^{2}} \operatorname{dist}^{2}\left(u(x), \mathbb{Z}^{N}\right) \mathrm{d} x .
\end{aligned}
$$

We prove that, up to subsequences in $\varepsilon$, there exists a density function $\varphi: \mathbb{Z}^{N} \times S^{1} \rightarrow[0, \infty)$ such that $F_{\varepsilon} \Gamma\left(L^{1}\left(T^{2}\right)\right)$-converges to

$$
F(u)=\int_{S_{u}} \varphi\left([u], n_{u}\right) \mathrm{d} \mathcal{H}^{1} .
$$

According to the variational model discussed in Section 2, the singular set $S_{u}$ of the phase-field $u$ represents dislocation line ensembles where the energy concentrates as $\varepsilon \rightarrow 0$. The density function $\varphi(s, n)$ estimates at every point $x_{0} \in S_{u}$ the anisotropic elastic interactions of the crystal, in terms of the normal vector $n=n_{u}\left(x_{0}\right)$ and the jump $s=[u]\left(x_{0}\right)$ across dislocation lines.

From now on for the reader's convenience we will use the following notation:

$$
\begin{aligned}
\mathbb{J}_{\varepsilon}[u](x, y) & :=\frac{1}{|\log \varepsilon|}(u(x)-u(y))^{T} \mathbb{J}(x-y)(u(x)-u(y)), \\
\mathbb{W}_{\varepsilon}[u](x) & :=\frac{1}{\varepsilon|\log \varepsilon|} \operatorname{dist}^{2}\left(u(x), \mathbb{Z}^{N}\right) .
\end{aligned}
$$

We also introduce the localization of $F_{\varepsilon}$ on the open sets $\mathcal{A}\left(T^{2}\right)$ in $T^{2}$ by

$$
F_{\varepsilon}(u, A):= \begin{cases}\int_{A \times A} \mathbb{J}_{\varepsilon}[u](x, y) \mathrm{d} x \mathrm{~d} y+\int_{A} \mathbb{W}_{\varepsilon}[u](x) \mathrm{d} x & \text { if } u \in H^{1 / 2}\left(T^{2}\right), \\ +\infty & \text { otherwise. }\end{cases}
$$

Note that the functional $F_{\varepsilon}$ is invariant with respect to integer translations, i.e.

$$
F_{\varepsilon}(u+c)=F_{\varepsilon}(u), \quad \forall c \in \mathbb{Z}^{N},
$$

and that the kernel $\mathbb{J}$ defines a quadratic form equivalent to the square of the $H^{1 / 2}\left(T^{2}\right)$ seminorm. In particular, $\mathbb{J}(x)=\mathcal{O}\left(|x|^{-3}\right)$ as $|x| \rightarrow 0$ and there exist constants $C_{1}, C_{2}>0$ such that

$$
\frac{C_{1}}{|x|^{3}}|v|^{2} \leqslant v^{T} \mathbb{J}(x) v \leqslant \frac{C_{2}}{|x|^{3}}|v|^{2}, \quad \forall v \in \mathbb{R}^{N} \text { and } \forall x \in T^{2} .
$$

The main result of this section is the following.

THEOREM 2 For every sequence $\left\{\varepsilon_{h}\right\}$ of positive real numbers such that $\varepsilon_{h} \rightarrow 0$ there exists a subsequence, denoted again by $\left\{\varepsilon_{h}\right\}$, such that the functionals $F_{\varepsilon_{h}}$ defined in $19, \Gamma\left(L^{1}\right)$-converge as $h \rightarrow \infty$. More precisely: 
(i) (Coerciveness) For every sequence $\left\{u_{h}\right\} \subseteq L^{1}\left(T^{2}\right)$ such that

$$
\sup _{h \in \mathbb{N}} F_{\varepsilon_{h}}\left(u_{h}, T^{2}\right)<\infty
$$

there exists a sequence $\left\{a_{h}\right\} \subset \mathbb{Z}^{N}$ such that $\left\{u_{h}-a_{h}\right\}$ is bounded in $L^{2}\left(T^{2}\right)$ and strongly pre-compact in $L^{1}\left(T^{2}\right)$; every cluster point of $\left\{u_{h}-a_{h}\right\}$ belongs to $B V\left(T^{2}, \mathbb{Z}^{N}\right)$.

(ii) ( $\Gamma$-convergence) There exists a density function $\varphi: \mathbb{Z}^{N} \times S^{1} \rightarrow[0, \infty)$ such that, as $h \rightarrow \infty$, the functional

$$
F(u, A)=\Gamma\left(L^{1}\right)-\lim _{h \rightarrow \infty} F_{\varepsilon_{h}}(u, A)
$$

exists for all $(u, A) \in L^{1}\left(T^{2}\right) \times \mathcal{A}\left(T^{2}\right)$ and can be written as

$$
F(u, A)= \begin{cases}\int_{S_{u} \cap A} \varphi\left([u], n_{u}\right) \mathrm{d} \mathcal{H}^{1} & \text { if } u \in B V\left(T^{2}, \mathbb{Z}^{N}\right), \\ +\infty & \text { otherwise. }\end{cases}
$$

In particular, for every $s \in \mathbb{Z}^{N}$ and $n \in S^{1}$ we have

$$
\varphi(s, n)=F\left(u_{s}^{n}, Q^{n}\right),
$$

where $Q^{n}$ is the unit square centered in the origin with a side parallel to $n$ and where $u_{s}^{n}$ is the step function defined by $u_{s}^{n}(x)=s \chi_{\{x \cdot n>0\}}$.

More precisely:

(a) ( $\Gamma$-liminf inequality) For every sequence $\left\{u_{h}\right\}$ converging to $u$ in $L^{1}\left(T^{2}\right)$ the following inequality holds:

$$
F(u, A) \leqslant \liminf _{h \rightarrow \infty} F_{\varepsilon_{h}}\left(u_{h}, A\right) .
$$

(b) (Recovery sequence) For every $u \in B V\left(T^{2}, \mathbb{Z}^{N}\right)$ there exists a sequence $\left\{u_{h}\right\}$ converging to $u$ in $L^{1}\left(T^{2}\right)$ such that

$$
F(u, A) \geqslant \limsup _{h \rightarrow \infty} F_{\varepsilon_{h}}\left(u_{h}, A\right) .
$$

REMARK 3 We remark that 20 is the only assumption on the kernel $\mathbb{J}$ we use in the proof of Theorem 2 . Hence our result can also be applied to different problems, e.g. different boundary conditions. Similarly the potential $\operatorname{dist}^{2}\left(\cdot, \mathbb{Z}^{N}\right)$ can be replaced by any other nonnegative function vanishing exactly on $\mathbb{Z}^{N}$ with some additional assumption on the behavior near the wells.

We will prove Theorem2 2 in a nonconstructive way (see [11, Chapter 16] for an overview of the so called localization method). For a given sequence $\varepsilon_{h} \rightarrow 0$ we define the $\Gamma\left(L^{1}\right)$-liminf and the $\Gamma\left(L^{1}\right)$-limsup of $F_{\varepsilon_{h}}$ respectively:

$$
\begin{aligned}
& F^{\prime}(u, A):=\inf \left\{\liminf _{h \rightarrow \infty} F_{\varepsilon_{h}}\left(u_{\varepsilon_{h}}, A\right): u_{\varepsilon_{h}} \stackrel{L^{1}}{\rightarrow} u\right\}, \\
& F^{\prime \prime}(u, A):=\inf \left\{\limsup _{h \rightarrow \infty} F_{\varepsilon_{h}}\left(u_{\varepsilon_{h}}, A\right): u_{\varepsilon_{h}} \stackrel{L^{1}}{\rightarrow} u\right\} .
\end{aligned}
$$

When the $\Gamma$-limit exists, it coincides with the $\Gamma$-limsup and the $\Gamma$-liminf. If the $\Gamma$-limit $F(u, A)$ exists for all open sets $A$, we extend it by inner regularity to any Borel subset $B \in \mathcal{B}\left(T^{2}\right)$, and we 
treat it as a set function for any given $u \in L^{1}\left(T^{2}\right)$. The aim is to prove that for any $\mathbb{Z}^{N}$-valued slip field $u, F(u, \cdot)$ is a measure with suitable properties that guarantee an integral representation of the type $(18)$.

In view of the coerciveness result we expect that the natural domain for the limit energy will be the space $B V\left(T^{2}, \mathbb{Z}^{N}\right)$, thus we will apply a well-known integral representation result for functionals defined on Caccioppoli partitions, due to Ambrosio and Braides, which we state below for the reader's convenience in a form suited to our case (see [6], [7] and [11] for details).

THEOREM 4 ([6]) Let $G: B V\left(T^{2}, \mathbb{Z}^{N}\right) \times \mathcal{B}\left(T^{2}\right) \rightarrow[0, \infty)$ be a functional satisfying the following assumptions:

(i) $G(u, \cdot)$ is a measure on $T^{2}$ for all $u \in B V\left(T^{2}, \mathbb{Z}^{N}\right)$;

(ii) $G$ is local on $\mathcal{A}\left(T^{2}\right)$, i.e., $G(u, A)=G(v, A)$ for all $A \in \mathcal{A}\left(T^{2}\right)$ and $u, v \in B V\left(T^{2}, \mathbb{Z}^{N}\right)$ such that $u=v$ a.e. on $A$;

(iii) $G(\cdot, A)$ is $L^{1}$-lower semicontinuous on $B V\left(T^{2}, \mathbb{Z}^{N}\right)$ for all $A \in \mathcal{A}\left(T^{2}\right)$;

(iv) there exist positive constants $c_{1}$ and $c_{2}$ such that, for all $u \in B V\left(T^{2}, \mathbb{Z}^{N}\right)$ and $B \in \mathcal{B}\left(T^{2}\right)$,

$$
c_{1}\left(\mathcal{H}^{n-1}\left(B \cap S_{u}\right)+|D u|(B)\right) \leqslant G(u, B) \leqslant c_{2}\left(\mathcal{H}^{n-1}\left(B \cap S_{u}\right)+|D u|(B)\right) .
$$

Then $G$ admits the following integral representation:

$$
G(u, B)=\int_{S_{u} \cap B} \varphi\left(x, u^{+}, u^{-}, n_{u}\right) \mathrm{d} \mathcal{H}^{1}, \quad u \in B V\left(T^{2}, \mathbb{Z}^{N}\right), B \in \mathcal{B}\left(T^{2}\right),
$$

with $\varphi: T^{2} \times \mathbb{Z}^{N} \times \mathbb{Z}^{N} \times S^{1} \rightarrow[0, \infty)$ defined by

$$
\varphi(x, i, j, n)=\limsup _{\rho \rightarrow 0^{+}} \frac{1}{\rho} \min \left\{G\left(u, \overline{Q_{\rho}^{n}}(x)\right): u \in \mathcal{X}\left(Q_{\rho}^{n}(x)\right)\right\}
$$

and

$$
\mathcal{X}\left(Q_{\rho}^{n}(x)\right)=\left\{u \in B V\left(T^{2}, \mathbb{Z}^{N}\right): u=u_{i j}^{n x} \text { on } T^{2} \backslash Q_{\rho}^{n}(x)\right\},
$$

where $Q_{\rho}^{n}(x)$ denotes the square centered at $x$ with side of length $\rho$ parallel to $n, \overline{Q_{\rho}^{n}}(x)$ its closure and $u_{i j}^{n x}: T^{2} \rightarrow \mathbb{Z}^{N}$ is the step function defined by

$$
u_{i j}^{n x}(y):= \begin{cases}i & \text { if }(y-x) \cdot n>0 \\ j & \text { if }(y-x) \cdot n \leqslant 0 .\end{cases}
$$

The proof of Theorem 2 follows from Section 3.1 where the coerciveness is easily derived by the scalar case, then from the Fundamental Estimate proved in Section 3.2 which permits one to prove the inner regularity of the $\Gamma$-limsup and $\Gamma$-liminf and hence existence of the $\Gamma$-limit up to a subsequence, and finally from an application of the representation theorem above in Section 3.3 .

\subsection{Coerciveness}

In this section we briefly deduce the coerciveness result stated in Theorem 2(i), as a consequence of the analogous result for the scalar case stated in Theorem 1 . 
Since

$$
\operatorname{dist}^{2}\left(u_{\varepsilon}, \mathbb{Z}^{N}\right)=\sum_{i=1}^{N} \operatorname{dist}^{2}\left(u_{\varepsilon}^{i}, \mathbb{Z}\right)
$$

by 20$)$ we get

$$
\begin{array}{r}
\min \left\{C_{1}, 1\right\} \sum_{i=1}^{N}\left(\frac{1}{|\log \varepsilon|} \int_{T^{2}} \int_{T^{2}} \frac{\left|u_{\varepsilon}^{i}(x)-u_{\varepsilon}^{i}(y)\right|^{2}}{|x-y|^{3}} \mathrm{~d} x \mathrm{~d} y+\frac{1}{\varepsilon|\log \varepsilon|} \int_{T^{2}} \operatorname{dist}^{2}\left(u_{\varepsilon}^{i}, \mathbb{Z}\right) \mathrm{d} x\right) \\
\leqslant F_{\varepsilon}\left(u_{\varepsilon}, T^{2}\right) .
\end{array}
$$

Then all components $u_{\varepsilon}^{i}$ of $u_{\varepsilon}$ satisfy

$$
\sup _{\varepsilon>0} I_{\varepsilon}\left(u_{\varepsilon}^{i}\right)<\infty \quad \text { for all } i=1, \ldots, N,
$$

where $I_{\varepsilon}$ is the scalar functional of dislocations $\left[15\right.$, corresponding to the choice $\mathrm{J}(x)=|x|^{-3}$ for the elastic interaction kernel.

By Theorem 1 for all $i=1, \ldots, N$ there exists a sequence $\left\{a_{\varepsilon}^{i}\right\} \subset \mathbb{Z}$ such that $\left\{u_{\varepsilon}^{i}-a_{\varepsilon}^{i}\right\}$ is bounded in $L^{2}\left(T^{2}, \mathbb{R}\right)$ and strongly pre-compact in $L^{1}\left(T^{2}, \mathbb{R}\right)$. Moreover, every cluster point of the translated sequence belongs to $B V\left(T^{2}, \mathbb{Z}\right)$. We conclude by setting $a_{\varepsilon}=\left(a_{\varepsilon}^{1}, \ldots, a_{\varepsilon}^{N}\right)$.

\subsection{Existence of the $\Gamma$-limit: the Fundamental Estimate}

In this section we establish the following compactness result.

THEOREM 5 Let $F_{\varepsilon}(u, A): L^{1}\left(T^{2}\right) \times \mathcal{A}\left(T^{2}\right) \rightarrow[0, \infty]$ be the functional defined in 19 . For every sequence $\left\{\varepsilon_{h}\right\}$ of positive real numbers such that $\varepsilon_{h} \rightarrow 0$ there exists a subsequence, denoted again by $\left\{\varepsilon_{h}\right\}$, such that the $\Gamma$-limit

$$
F(u, A)=\Gamma\left(L^{1}\right)-\lim _{h \rightarrow \infty} F_{\varepsilon_{h}}(u, A)
$$

exists for all $(u, A) \in L^{1}\left(T^{2}\right) \times \mathcal{A}\left(T^{2}\right)$.

For a fixed a sequence $\varepsilon_{h} \rightarrow 0$, we begin by considering the $\Gamma\left(L^{1}\right)$-liminf $F^{\prime}(u, A)$ and the $\Gamma\left(L^{1}\right)$-limsup $F^{\prime \prime}(u, A)$ defined in 23 and 24 . Since the functional $F_{\varepsilon_{h}}$ is nonnegative, it follows that for all $u \in L^{1}\left(T^{2}\right)$, the functions $F^{\prime}(u, \cdot), F^{\prime \prime}(u, \cdot)$ are increasing set functions:

$$
F^{\prime}(u, A) \leqslant F^{\prime}(u, B), \quad F^{\prime \prime}(u, A) \leqslant F^{\prime \prime}(u, B), \quad \forall A, B \in \mathcal{A}\left(T^{2}\right): A \subseteq B .
$$

The main step is to prove that $F^{\prime}$ and $F^{\prime \prime}$ have the inner regularity property:

$$
\begin{aligned}
F^{\prime}(u, A) & =\sup \left\{F^{\prime}\left(u, A^{\prime}\right): A^{\prime} \in \mathcal{A}\left(T^{2}\right), A^{\prime} \subset \subset A\right\}, \\
F^{\prime \prime}(u, A) & =\sup \left\{F^{\prime \prime}\left(u, A^{\prime}\right): A^{\prime} \in \mathcal{A}\left(T^{2}\right), A^{\prime} \subset \subset A\right\} .
\end{aligned}
$$

This property is ensured by the Fundamental Estimate for the functional $F_{\varepsilon}$, contained in the next theorem. In what follows, by a cut-off function between $A^{\prime}$ and $A$, with $A, A^{\prime} \in \mathcal{A}\left(T^{2}\right)$ and $A^{\prime} \subset \subset A$, we mean a function $\varphi: A \rightarrow \mathbb{R}$ such that $\varphi \in C_{0}^{\infty}(A), 0 \leqslant \varphi \leqslant 1$ and $\varphi \equiv 1$ on $A^{\prime}$. 
Theorem 6 (The Fundamental Estimate) For any $A, A^{\prime}, B \in \mathcal{A}\left(T^{2}\right)$ with $A^{\prime} \subset \subset A$ and every $\sigma>0$, there exists a positive constant $C_{\sigma}=C\left(\sigma, A, A^{\prime}, B\right)$ such that, for all $u, v \in L^{1}\left(T^{2}\right)$, there exists a cut-off function $\varphi$ between $A^{\prime}$ and $A$ such that

$$
\begin{aligned}
& F_{\varepsilon}\left(\varphi u+(1-\varphi) v, A^{\prime} \cup B\right) \\
& \qquad(1+\sigma)\left(F_{\varepsilon}(u, A)+F_{\varepsilon}(v, B)\right)+\frac{C_{\sigma}}{|\log \varepsilon|}\left(\int_{A}|u|^{2} \mathrm{~d} x+\int_{B}|v|^{2} \mathrm{~d} x\right) .
\end{aligned}
$$

Proof. By the definition of $F_{\varepsilon}$ it is enough to consider pairs of functions $u, v \in H^{1 / 2}\left(T^{2}\right)$, otherwise 30 holds trivially.

We set $\delta=\operatorname{dist}\left(A^{\prime}, \partial A\right)$ and for any given $n \in \mathbb{N}$ we define the following sets:

$$
A_{0}=A^{\prime}, \quad A_{k}=\left\{x \in A: \operatorname{dist}\left(x, A^{\prime}\right)<k \delta / n\right\}, \quad k \in\{1, \ldots, n\} .
$$

For any fixed $k \in\{1, \ldots, n\}$ let $\varphi \equiv \varphi_{k}$ be a cut-off function between $A_{k-1}$ and $A_{k}$ such that $|\nabla \varphi| \leqslant n / \delta$. We set

$$
\begin{gathered}
C_{i}=\left(A_{i} \backslash A_{i-1}\right) \cap B, \quad i=1, \ldots, n, \\
U=A_{0} \cup \bigcup_{i=1}^{k-1} C_{i}, \quad V=B \backslash A_{k} .
\end{gathered}
$$

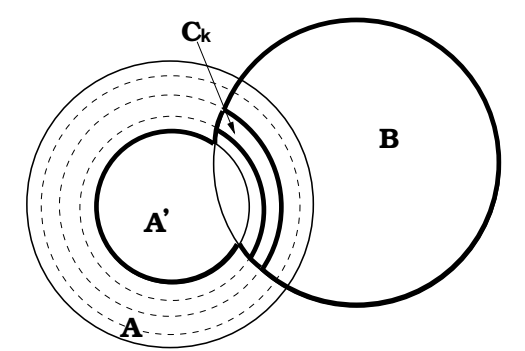

FIG. 1. Choice of a good slice for the cut-off function.

It follows that $\left\{U, C_{k}, V\right\}$ is a partition of $A^{\prime} \cup B$, as shown in Figure 1 . Then we set $w:=\varphi u+$ $(1-\varphi) v$ and we compute its energy in $A^{\prime} \cup B$ :

$$
\begin{aligned}
F_{\varepsilon}\left(w, A^{\prime} \cup B\right)= & \int_{A^{\prime} \cup B} \int_{A^{\prime} \cup B} \mathbb{J}_{\varepsilon}[w](x, y) \mathrm{d} x \mathrm{~d} y+\int_{A^{\prime} \cup B} \mathbb{W}_{\varepsilon}[w](x) \mathrm{d} x \\
\leqslant & \int_{U} \int_{U} \mathbb{J}_{\varepsilon}[w](x, y) \mathrm{d} x \mathrm{~d} y+\int_{C_{k}} \int_{C_{k}} \mathbb{J}_{\varepsilon}[w](x, y) \mathrm{d} x \mathrm{~d} y \\
& +\int_{V} \int_{V} \mathbb{J}_{\varepsilon}[w](x, y) \mathrm{d} x \mathrm{~d} y+2 \int_{U} \int_{C_{k}} \mathbb{J}_{\varepsilon}[w](x, y) \mathrm{d} x \mathrm{~d} y \\
& +2 \int_{V} \int_{C_{k}} \mathbb{J}_{\varepsilon}[w](x, y) \mathrm{d} x \mathrm{~d} y+2 \int_{U} \int_{V} \mathbb{J}_{\varepsilon}[w](x, y) \mathrm{d} x \mathrm{~d} y \\
& +\int_{A} \mathbb{W}_{\varepsilon}[w](x) \mathrm{d} x+\int_{B} \mathbb{W}_{\varepsilon}[w](x) \mathrm{d} x .
\end{aligned}
$$


By the definition of $\varphi$ it follows that $w=u$ on $U, w=v$ on $V$ and $U \subset A, V \subset B$, and hence

$$
\begin{aligned}
& F_{\varepsilon}\left(w, A^{\prime} \cup B\right) \leqslant F_{\varepsilon}(u, A)+F_{\varepsilon}(v, B)+\int_{C_{k}} \int_{C_{k}} \mathbb{J}_{\varepsilon}[w](x, y) \mathrm{d} x \mathrm{~d} y \\
& +2 \int_{U} \int_{V} \mathbb{J}_{\varepsilon}[w](x, y) \mathrm{d} x \mathrm{~d} y+2 \int_{U} \int_{C_{k}} \mathbb{J}_{\varepsilon}[w](x, y) \mathrm{d} x \mathrm{~d} y+2 \int_{V} \int_{C_{k}} \mathbb{J}_{\varepsilon}[w](x, y) \mathrm{d} x \mathrm{~d} y .
\end{aligned}
$$

By (20) the four double integrals in 31 can be controlled respectively by

$$
\begin{array}{ll}
I_{1}:=\frac{C_{2}}{|\log \varepsilon|} \int_{C_{k}} \int_{C_{k}} \frac{|w(x)-w(y)|^{2}}{|x-y|^{3}} \mathrm{~d} x \mathrm{~d} y, \quad I_{2}:=\frac{2 C_{2}}{|\log \varepsilon|} \int_{U} \int_{V} \frac{|w(x)-w(y)|^{2}}{|x-y|^{3}} \mathrm{~d} x \mathrm{~d} y, \\
I_{3}:=\frac{2 C_{2}}{|\log \varepsilon|} \int_{U} \int_{C_{k}} \frac{|w(x)-w(y)|^{2}}{|x-y|^{3}} \mathrm{~d} x \mathrm{~d} y, & I_{4}:=\frac{2 C_{2}}{|\log \varepsilon|} \int_{V} \int_{C_{k}} \frac{|w(x)-w(y)|^{2}}{|x-y|^{3}} \mathrm{~d} x \mathrm{~d} y .
\end{array}
$$

We evaluate the differences $|w(x)-w(y)|^{2}$ in each integral. For every $(x, y) \in C_{k} \times C_{k}$ it follows that

$$
\begin{aligned}
\mid w(x) & -\left.w(y)\right|^{2}=\sum_{i=1}^{N}\left|w^{i}(x)-w^{i}(y)\right|^{2} \\
& =\sum_{i=1}^{N}\left|\varphi(x) u^{i}(x)+(1-\varphi(x)) v^{i}(x)-\varphi(y) u^{i}(y)-(1-\varphi(y)) v^{i}(y)\right|^{2} \\
& =\sum_{i=1}^{N}\left|\varphi(x)\left(u^{i}(x)-u^{i}(y)\right)+(1-\varphi(x))\left(v^{i}(x)-v^{i}(y)\right)+(\varphi(x)-\varphi(y))\left(u^{i}(y)-v^{i}(y)\right)\right|^{2} \\
& \leqslant C \sum_{i=1}^{N}\left(\left|u^{i}(x)-u^{i}(y)\right|^{2}+\left|v^{i}(x)-v^{i}(y)\right|^{2}+|\varphi(x)-\varphi(y)|^{2}\left|u^{i}(y)-v^{i}(y)\right|^{2}\right),
\end{aligned}
$$

and then

$I_{1} \leqslant \frac{C}{|\log \varepsilon|} \int_{C_{k}} \int_{C_{k}}\left(\frac{|u(x)-u(y)|^{2}}{|x-y|^{3}}+\frac{|v(x)-v(y)|^{2}}{|x-y|^{3}}+\frac{|\varphi(x)-\varphi(y)|^{2}}{|x-y|^{3}}\left(|u(y)|^{2}+|v(y)|^{2}\right)\right) \mathrm{d} x \mathrm{~d} y$.

For every $(x, y) \in U \times V$ we have

$$
|w(x)-w(y)|^{2}=\sum_{i=1}^{N}\left|u^{i}(x)-v^{i}(y)\right|^{2} \leqslant C\left(|u(x)|^{2}+|v(y)|^{2}\right),
$$

and

$$
I_{2} \leqslant \frac{C}{|\log \varepsilon|} \int_{U} \int_{V} \frac{|u(x)|^{2}+|v(y)|^{2}}{|x-y|^{3}} \mathrm{~d} x \mathrm{~d} y .
$$


For every $(x, y) \in U \times C_{k}$ we have

$$
\begin{aligned}
|w(x)-w(y)|^{2} & =\sum_{i=1}^{N}\left|u^{i}(x)-\varphi(y) u^{i}(y)-(1-\varphi(y)) v^{i}(y)\right|^{2} \\
& =\sum_{i=1}^{N}\left|\left(u^{i}(x)-u^{i}(y)\right)+(1-\varphi(y))\left(u^{i}(y)-v^{i}(y)\right)\right|^{2} \\
& \leqslant C \sum_{i=1}^{N}\left(\left|u^{i}(x)-u^{i}(y)\right|^{2}+|\varphi(x)-\varphi(y)|^{2}\left|u^{i}(y)-v^{i}(y)\right|^{2}\right)
\end{aligned}
$$

and

$$
I_{3} \leqslant \frac{C}{|\log \varepsilon|} \int_{U} \int_{C_{k}}\left(\frac{|u(x)-u(y)|^{2}}{|x-y|^{3}}+\frac{|\varphi(x)-\varphi(y)|^{2}}{|x-y|^{3}}\left(|u(y)|^{2}+|v(y)|^{2}\right)\right) \mathrm{d} x \mathrm{~d} y .
$$

Similarly for every $(x, y) \in V \times C_{k}$ it follows that

$$
I_{4} \leqslant \frac{C}{|\log \varepsilon|} \int_{V} \int_{C_{k}}\left(\frac{|v(x)-v(y)|^{2}}{|x-y|^{3}}+\frac{|\varphi(x)-\varphi(y)|^{2}}{|x-y|^{3}}\left(|u(y)|^{2}+|v(y)|^{2}\right)\right) \mathrm{d} x \mathrm{~d} y .
$$

We set $I:=I_{1}+I_{2}+I_{3}+I_{4}$ and from $32-35$ we get

$$
\begin{aligned}
I \leqslant & \frac{C}{|\log \varepsilon|}\left\{\int_{U \cup C_{k}} \int_{C_{k}} \frac{|u(x)-u(y)|^{2}}{|x-y|^{3}} \mathrm{~d} x \mathrm{~d} y+\int_{V \cup C_{k}} \int_{C_{k}} \frac{|v(x)-v(y)|^{2}}{|x-y|^{3}} \mathrm{~d} x \mathrm{~d} y\right. \\
& \left.+\int_{A^{\prime} \cup B} \int_{C_{k}}|\varphi(x)-\varphi(y)|^{2} \frac{|u(y)|^{2}+|v(y)|^{2}}{|x-y|^{3}} \mathrm{~d} x \mathrm{~d} y+\int_{U} \int_{V} \frac{|u(x)|^{2}+|v(y)|^{2}}{|x-y|^{3}} \mathrm{~d} x \mathrm{~d} y\right\} .
\end{aligned}
$$

Again by 20] and from the fact that $U \cup C_{k} \subset A, V \cup C_{k} \subset B$, we conclude that

$$
\begin{aligned}
I \leqslant & C\left(\int_{A} \int_{C_{k}} \mathbb{J}_{\varepsilon}[u](x, y) \mathrm{d} x \mathrm{~d} y+\int_{B} \int_{C_{k}} \mathbb{J}_{\varepsilon}[v](x, y) \mathrm{d} x \mathrm{~d} y\right) \\
& +\frac{C}{|\log \varepsilon|} \int_{A^{\prime} \cup B} \int_{C_{k}}|\varphi(x)-\varphi(y)|^{2} \frac{|u(y)|^{2}+|v(y)|^{2}}{|x-y|^{3}} \mathrm{~d} x \mathrm{~d} y \\
& +\frac{C}{|\log \varepsilon|} \int_{U} \int_{V} \frac{|u(x)|^{2}+|v(y)|^{2}}{|x-y|^{3}} \mathrm{~d} x \mathrm{~d} y .
\end{aligned}
$$

We now remark that

$$
\begin{aligned}
\sum_{k=1}^{n}\left(\int_{A} \int_{C_{k}} \mathbb{J}_{\varepsilon}[u](x, y) \mathrm{d} x \mathrm{~d} y+\int_{B} \int_{C_{k}} \mathbb{J}_{\varepsilon}[v](x, y) \mathrm{d} x \mathrm{~d} y\right) \\
\quad \leqslant \int_{A} \int_{A} \mathbb{J}_{\varepsilon}[u](x, y) \mathrm{d} x \mathrm{~d} y+\int_{B} \int_{B} \mathbb{J}_{\varepsilon}[v](x, y) \mathrm{d} x \mathrm{~d} y \leqslant F_{\varepsilon}(u, A)+F_{\varepsilon}(v, B) .
\end{aligned}
$$

Then we can choose $k \in\{1, \ldots, n\}$ such that

$$
\int_{A} \int_{C_{k}} \mathbb{J}_{\varepsilon}[u](x, y) \mathrm{d} x \mathrm{~d} y+\int_{B} \int_{C_{k}} \mathbb{J}_{\varepsilon}[v](x, y) \mathrm{d} x \mathrm{~d} y \leqslant \frac{1}{n}\left(F_{\varepsilon}(u, A)+F_{\varepsilon}(v, B)\right) .
$$


On the other hand, the cut-off function $\varphi$ satisfies

$$
|\varphi(x)-\varphi(y)| \leqslant \frac{n}{\delta}|x-y|
$$

by construction and

$$
\int_{A^{\prime} \cup B} \frac{\mathrm{d} x}{|x-y|} \stackrel{[z=x-y]}{=} \int_{\left(A^{\prime} \cup B\right)-y} \frac{\mathrm{d} z}{|z|} \leqslant \int_{D} \frac{\mathrm{d} z}{|z|} \leqslant C,
$$

where $D$ is a disc containing the set $\left(A^{\prime} \cup B\right)-y$ for all $y \in C_{k}$. Therefore it follows that

$$
\begin{aligned}
\int_{A^{\prime} \cup B} \int_{C_{k}} \frac{|\varphi(x)-\varphi(y)|^{2}}{|x-y|^{3}}\left(|u(y)|^{2}\right. & \left.+|v(y)|^{2}\right) \mathrm{d} x \mathrm{~d} y \\
& \leqslant\left(\frac{n}{\delta}\right)^{2} \int_{C_{k}}\left(\int_{A^{\prime} \cup B} \frac{\mathrm{d} x}{|x-y|}\right)\left(|u(y)|^{2}+|v(y)|^{2}\right) \mathrm{d} y \\
& \leqslant C\left(\frac{n}{\delta}\right)^{2} \int_{C_{k}}\left(|u(y)|^{2}+|v(y)|^{2}\right) \mathrm{d} y .
\end{aligned}
$$

Finally, for every $(x, y) \in U \times V$ we have $|x-y|>\delta / n$ and

$$
\begin{aligned}
& \int_{U} \int_{V} \frac{|u(x)|^{2}+|v(y)|^{2}}{|x-y|^{3}} \mathrm{~d} x \mathrm{~d} y \leqslant\left(\frac{n}{\delta}\right)^{3}\left(|V| \int_{U}|u(x)|^{2} \mathrm{~d} x+|U| \int_{V}|v(y)|^{2} \mathrm{~d} y\right) \\
& \leqslant \max \{|A|,|B|\}\left(\frac{n}{\delta}\right)^{3}\left(\int_{U}|u(x)|^{2} \mathrm{~d} x+\int_{V}|v(y)|^{2} \mathrm{~d} y\right) .
\end{aligned}
$$

We now set

$$
\bar{C}=\bar{C}\left(n, A, A^{\prime}, B\right)=C \max \left\{(n / \delta)^{2}, \max \{|A|,|B|\}(n / \delta)^{3}\right\} .
$$

and from 31 and $36-39$ we conclude that

$$
\begin{aligned}
& F_{\varepsilon}\left(\varphi u+(1-\varphi) v, A^{\prime} \cup B\right) \\
& \quad \leqslant\left(1+\frac{C}{n}\right)\left(F_{\varepsilon}(u, A)+F_{\varepsilon}(v, B)\right)+\frac{\bar{C}}{|\log \varepsilon|}\left(\int_{U \cup C_{k}}|u|^{2} \mathrm{~d} x+\int_{V \cup C_{k}}|v|^{2} \mathrm{~d} x\right) .
\end{aligned}
$$

Now choose $n=n_{\sigma}:=[C / \sigma]+1$ and set $C_{\sigma}:=\bar{C}\left(n_{\sigma}, A, A^{\prime}, B\right)$. Since $U \cup C_{k} \subset A$ and $V \cup C_{k} \subset B$ it follows that

$F_{\varepsilon}\left(\varphi u+(1-\varphi) v, A^{\prime} \cup B\right) \leqslant(1+\sigma)\left(F_{\varepsilon}(u, A)+F_{\varepsilon}(v, B)\right)+\frac{C_{\sigma}}{|\log \varepsilon|}\left(\int_{A}|u|^{2} \mathrm{~d} x+\int_{B}|v|^{2} \mathrm{~d} x\right)$, and this finishes the proof.

We now prove the inner regularity property of the $\Gamma$-liminf and the $\Gamma$-limsup of $F_{\varepsilon}$.

Proposition 7 For every $u \in L^{1}\left(T^{2}\right)$ the set functions $F^{\prime}(u, \cdot), F^{\prime \prime}(u, \cdot): \mathcal{A}\left(T^{2}\right) \rightarrow[0, \infty]$ defined in 23) and (24) have the inner regularity property [29]. 
Proof. By the coercivity property of $F_{\varepsilon}$ it is enough to consider functions $u \in B V\left(T^{2}, \mathbb{Z}^{N}\right)$. For every sequence $\left\{\varepsilon_{h}\right\}$ of positive real numbers such that $\varepsilon_{h} \rightarrow 0$ and every $A, B \in \mathcal{A}\left(T^{2}\right)$, let $\left\{u_{h}\right\},\left\{v_{h}\right\} \subset L^{1}\left(T^{2}\right)$ be recovery sequences for $F^{\prime}(u, A)$ and $F^{\prime \prime}(u, B)$ respectively, i.e.,

$$
\begin{aligned}
& u_{h} \stackrel{h \rightarrow \infty}{\longrightarrow} u \text { in } L^{1}\left(T^{2}\right), \quad F^{\prime}(u, A)=\liminf _{h \rightarrow \infty} F_{\varepsilon_{h}}\left(u_{h}, A\right), \\
& v_{n} \stackrel{h \rightarrow \infty}{\longrightarrow} u \text { in } L^{1}\left(T^{2}\right), \quad F^{\prime \prime}(u, B)=\limsup _{h \rightarrow \infty} F_{\varepsilon_{h}}\left(v_{h}, B\right) .
\end{aligned}
$$

For all $h \in \mathbb{N}$ we apply the fundamental estimate 30 to $u_{h}$ and $v_{h}$ : for every $A^{\prime} \in \mathcal{A}\left(T^{2}\right)$ with $A^{\prime} \subset \subset A$ and every $\sigma>0$ there exists a constant $C_{\sigma}>0$ such that

$$
\begin{aligned}
& F_{\varepsilon_{h}}\left(\varphi_{h} u_{h}+\left(1-\varphi_{h}\right) v_{h}, A^{\prime} \cup B\right) \\
& \quad \leqslant(1+\sigma)\left(F_{\varepsilon_{h}}\left(u_{h}, A\right)+F_{\varepsilon_{h}}\left(v_{h}, B\right)\right)+\frac{C_{\sigma}}{\left|\log \varepsilon_{h}\right|}\left(\int_{A}\left|u_{h}\right|^{2}+\int_{B}\left|v_{h}\right|^{2}\right),
\end{aligned}
$$

where $\left\{\varphi_{h}\right\}$ is a suitable sequence of cut-off functions between $A^{\prime}$ and $A$. Moreover,

$$
\left|\varphi_{h} u_{h}+\left(1-\varphi_{h}\right) v_{h}-u\right| \leqslant\left|\varphi_{h}\right|\left|u_{h}-u\right|+\left|1-\varphi_{h}\right|\left|v_{h}-u\right| \leqslant\left|u_{h}-u\right|+\left|v_{h}-u\right|,
$$

i.e., $\varphi_{h} u_{h}+\left(1-\varphi_{h}\right) v_{h}$ also converges to $u$ in $L^{1}\left(T^{2}\right)$. Again by the coercivity property we may assume that the sequences $\left\{u_{h}\right\}$ and $\left\{v_{h}\right\}$ are bounded in $L^{2}\left(T^{2}\right)$. Taking the limit in 40 as $h \rightarrow \infty$ we get

$$
\begin{aligned}
F^{\prime}\left(u, A^{\prime} \cup B\right) & \leqslant \liminf _{h \rightarrow \infty} F_{\varepsilon_{h}}\left(\varphi_{h} u_{h}+\left(1-\varphi_{h}\right) v_{h}, A^{\prime} \cup B\right) \\
& \leqslant(1+\sigma)\left(\liminf _{h \rightarrow \infty} F_{\varepsilon_{h}}\left(u_{h}, A\right)+\limsup _{h \rightarrow \infty} F_{\varepsilon_{h}}\left(v_{h}, B\right)\right) \\
& \leqslant(1+\sigma)\left(F^{\prime}(u, A)+F^{\prime \prime}(u, B)\right)
\end{aligned}
$$

for all $\sigma>0$, where the first inequality holds by the definition of $F^{\prime}$. By the arbitrariness of $\sigma$ we conclude

$$
F^{\prime}\left(u, A^{\prime} \cup B\right) \leqslant F^{\prime}(u, A)+F^{\prime \prime}(u, B) .
$$

Similarly we get

$$
F^{\prime \prime}\left(u, A^{\prime} \cup B\right) \leqslant F^{\prime \prime}(u, A)+F^{\prime \prime}(u, B) .
$$

Since $F^{\prime}$ and $F^{\prime \prime}$ are increasing set functions, for every $W, A \in \mathcal{A}\left(T^{2}\right)$ with $A \subset \subset W$ it follows that

$$
F^{\prime}(u, W) \geqslant \sup \left\{F^{\prime}(u, A): A \subset \subset W\right\}, \quad F^{\prime \prime}(u, W) \geqslant \sup \left\{F^{\prime \prime}(u, A): A \subset \subset W\right\} .
$$

In order to prove the inner regularity property we have to prove the opposite inequalities. Consider a compact set $K$ and $A^{\prime}, A, W \in \mathcal{A}\left(T^{2}\right)$ such that $K \subset \subset A^{\prime} \subset \subset A \subset \subset W$. We apply (41) and (42) with $B=W \backslash K$ :

$$
F^{\prime}(u, W) \leqslant F^{\prime}(u, A)+F^{\prime \prime}(u, W \backslash K), \quad F^{\prime \prime}(u, W) \leqslant F^{\prime \prime}(u, A)+F^{\prime \prime}(u, W \backslash K) .
$$

Taking the supremum over all open sets $A \subset \subset W$, it follows that

$$
\begin{gathered}
F^{\prime}(u, W) \leqslant \sup \left\{F^{\prime}(u, A): A \subset \subset W\right\}+F^{\prime \prime}(u, W \backslash K), \\
F^{\prime \prime}(u, W) \leqslant \sup \left\{F^{\prime \prime}(u, A): A \subset \subset W\right\}+F^{\prime \prime}(u, W \backslash K) .
\end{gathered}
$$


We now prove that the term $F^{\prime \prime}(u, W \backslash K)$ vanishes as $K$ grows in $W$. From the fact that the functional $F_{\varepsilon}$ is nonnegative and by 20$\}$, for every sequence $\left\{u_{h}\right\}$ converging to $u$ in $L^{1}\left(T^{2}\right)$, we get

$$
0 \leqslant F_{\varepsilon_{h}}\left(u_{h}, W \backslash K\right) \leqslant C \sum_{i=1}^{N} I_{\varepsilon_{h}}\left(u_{h}^{i}, W \backslash K\right),
$$

where $I_{\varepsilon_{h}}\left(u_{h}^{i}, W \backslash K\right)$ is the localization over $W \backslash K$ of the scalar functional of dislocations $\sqrt{15}$, evaluated on the $i$ th component of $\left\{u_{h}\right\}$ and corresponding to the choice $\mathrm{J}(x)=|x|^{-3}$. By Theorem 1 the functional $I_{\varepsilon_{h}} \Gamma\left(L^{1}\right)$-converges to

$$
I(v, W \backslash K)=\int_{S_{v} \cap(W \backslash K)} \gamma(n)|[v]| \mathrm{d} \mathcal{H}^{1}, \quad \forall v \in B V\left(T^{2}, \mathbb{Z}\right),
$$

where, for every $n \in S^{1}$,

$$
\gamma(n)=2 \int_{x \cdot n=1}|x|^{-3} \mathrm{~d} \mathcal{H}^{1}(x)=2 \int_{\mathbb{R}}\left(1+x_{2}^{2}\right)^{-3 / 2} \mathrm{~d} x_{2}=4\left[x_{2}\left(1+x_{2}^{2}\right)^{-1 / 2}\right]_{0}^{\infty}=4 .
$$

We choose $\left\{u_{h}\right\}$ such that for all $i=1, \ldots, N$, the sequence $\left\{u_{h}^{i}\right\}$ is a recovery sequence for $I\left(u^{i}, W \backslash K\right)$. Then, taking the limit in (44) as $h \rightarrow \infty$, we conclude that

$$
\begin{aligned}
0 & \leqslant F^{\prime \prime}(u, W \backslash K) \leqslant \limsup _{h \rightarrow \infty} F_{\varepsilon_{h}}\left(u_{h}, W \backslash K\right) \leqslant C \sum_{i=1}^{N} \int_{S_{u^{i}} \cap(W \backslash K)}\left|\left[u^{i}\right]\right| \mathrm{d} \mathcal{H}^{1} \\
& \leqslant C|D u|(W \backslash K),
\end{aligned}
$$

where the second inequality holds by definition of $F^{\prime \prime}$. Finally, we take the supremum over all compact sets $K \subset W$, so that the last term in (45) goes to zero. The proof is complete.

The existence of the $\Gamma$-limit of the functional of dislocations $F_{\varepsilon}$ is now a simple consequence of Proposition 7

Proof of Theorem 5 . We denote by $\mathcal{R}\left(T^{2}\right)$ the family of all finite unions of rectangles of $T^{2}$ whose vertices have rational coordinates.

By the compactness of the $\Gamma$-convergence and a diagonal argument, we can extract a subsequence from $\left\{\varepsilon_{h}\right\}$, again denoted by $\left\{\varepsilon_{h}\right\}$, such that the limit

$$
F(u, R)=\Gamma\left(L^{1}\right)-\lim _{h \rightarrow \infty} F_{\varepsilon_{h}}(u, R)=F^{\prime}(u, R)=F^{\prime \prime}(u, R)
$$

exists for all $u \in L^{1}\left(T^{2}\right)$ and $R \in \mathcal{R}\left(T^{2}\right)$. Using the inner regularity property stated in Proposition 7 we can show that this limit also exists for all open sets. Indeed, for every $A^{\prime}, A \in \mathcal{A}\left(T^{2}\right)$ with $A^{\prime} \subset \subset A$ there exists an open set $R \in \mathcal{R}\left(T^{2}\right)$ such that $A^{\prime} \subset \subset R \subset \subset A$. Then, by inner regularity of $F^{\prime}$ and $F^{\prime \prime}$, it follows that

$$
\begin{aligned}
F^{\prime}(u, A) & =\sup \left\{F^{\prime}\left(u, A^{\prime}\right): A^{\prime} \subset \subset A\right\}=\sup \left\{F^{\prime}(u, R): R \subset \subset A\right\} \\
& =\sup \left\{F^{\prime \prime}(u, R): R \subset \subset A\right\}=\sup \left\{F^{\prime \prime}\left(u, A^{\prime}\right): A^{\prime} \subset \subset A\right\}=F^{\prime \prime}(u, A),
\end{aligned}
$$

i.e.

$$
F(u, A):=F^{\prime}(u, A)=F^{\prime \prime}(u, A), \quad \forall(u, A) \in L^{1}\left(T^{2}\right) \times \mathcal{A}\left(T^{2}\right),
$$

which concludes the proof of the existence of the $\Gamma$-limit. 


\subsection{Integral representation}

In this section we complete the proof of Theorem 2, i.e., we obtain the integral representation (21) for the $\Gamma$-limit $F$ constructed in the previous section. We apply the integral representation result by Ambrosio and Braides (Theorem 4 ) and use the properties of our functional $F_{\varepsilon}$ to simplify the general formula [26] for the energy density $\varphi$.

THEOREM 8 The $\Gamma\left(L^{1}\right)$-limit $F$ obtained in 28 admits, for every $u \in B V\left(T^{2}, \mathbb{Z}^{N}\right)$ and $B \in$ $\mathcal{B}\left(T^{2}\right)$, the following integral representation:

$$
F(u, B)=\int_{S_{u} \cap B} \varphi\left([u], n_{u}\right) \mathrm{d} \mathcal{H}^{1} .
$$

The energy density function $\varphi: \mathbb{Z}^{N} \times S^{1} \rightarrow[0, \infty)$ is given by

$$
\varphi(s, n)=F\left(u_{s}^{n}, Q^{n}\right),
$$

where $Q^{n}$ is the unit square centered at the origin with a side parallel to $n$ and $u_{s}^{n}(x)=s \chi\{x \cdot n>0\}$.

Proof. We have to verify that the functional $F$ has properties (i)-(iv) of Theorem 4

We first remark that, by definition, $F$ inherits from $F^{\prime}$ and $F^{\prime \prime}$ the inner regularity property (Proposition 7). In particular, replacing $F^{\prime \prime}$ with $F$ in 42 , it follows that

$$
F\left(u, A^{\prime} \cup B\right) \leqslant F(u, A)+F(u, B) .
$$

Taking the supremum over all $A^{\prime} \in \mathcal{A}\left(T^{2}\right)$ such that $A^{\prime} \subset \subset A$, we obtain that $F$ is sub-additive.

We now prove that $F$ is also superadditive. For every $A, B \in \mathcal{A}\left(T^{2}\right)$ with $A \cap B=\emptyset$, let $\left\{u_{h}\right\}$ be a recovery sequence for $F(u, A \cup B)$. We have

$$
\begin{aligned}
F(u, A \cup B) & =\lim _{h \rightarrow \infty} F_{\varepsilon_{h}}\left(u_{h}, A \cup B\right) \\
& =\lim _{h \rightarrow \infty}\left(\int_{A \cup B} \int_{A \cup B} \mathbb{J}_{\varepsilon_{h}}\left[u_{h}\right](x, y) \mathrm{d} x \mathrm{~d} y+\int_{A \cup B} \mathbb{W}_{\varepsilon_{h}}\left[u_{h}\right](x) \mathrm{d} x\right) \\
& =\lim _{h \rightarrow \infty}\left(F_{\varepsilon_{h}}\left(u_{h}, A\right)+F_{\varepsilon_{h}}\left(u_{h}, B\right)+2 \int_{A} \int_{B} \mathbb{J}_{\varepsilon_{h}}\left[u_{h}\right](x, y) \mathrm{d} x \mathrm{~d} y\right) \\
& \geqslant \liminf _{h \rightarrow \infty} F_{\varepsilon_{h}}\left(u_{h}, A\right)+\liminf _{h \rightarrow \infty} F_{\varepsilon_{h}}\left(u_{h}, B\right) \geqslant F(u, A)+F(u, B),
\end{aligned}
$$

where we used the positivity of the quadratic form $\mathbb{J}_{\varepsilon_{h}}$ and the $\Gamma$-liminf inequality for the functional $F_{\varepsilon_{h}}$.

By the characterization of measures as increasing, subadditive, superadditive and inner-regular set functions (see the well known result by De Giorgi and Letta [13]), we deduce that $F(u, \cdot)$ is a Borel measure on $T^{2}$, i.e., property (i) of Theorem 4 holds.

On the other hand, properties (ii) and (iii) of Theorem 4 directly follow from the definition of $F(u, A)$; in particular, it is known that each $\Gamma\left(L^{1}\right)$-limit is always lower semicontinuous with respect to the $L^{1}$ topology.

Finally, by the estimate 20 on the kernel $\mathbb{J}$ we get (as in 44 ) and 45 )

$$
C_{1}|D u|(A) \leqslant F(u, A) \leqslant C_{2}|D u|(A) .
$$


Since $u \in B V\left(T^{2}, \mathbb{Z}^{N}\right)$ we have $|D u|(A)=\int_{A \cap S_{u}}|[u]| \mathrm{d} \mathcal{H}^{1}$ and $|[u]| \geqslant 1 \mathcal{H}^{1}$-a.e. on $S_{u}$. Then

$$
\frac{C_{1}}{2}\left(\mathcal{H}^{1}\left(A \cap S_{u}\right)+|D u|(A)\right) \leqslant F(u, A) \leqslant C_{2}\left(\mathcal{H}^{1}\left(A \cap S_{u}\right)+|D u|(A)\right),
$$

yielding property (iv) of Theorem 4 restricted to $\mathcal{A}\left(T^{2}\right)$, which can be exended to $\mathcal{B}\left(T^{2}\right)$ using the fact that $F(u, \cdot)$ is actually a measure.

Applying Theorem 4 we then obtain the following integral representation for the functional $F$ :

$$
F(u, B)=\int_{S_{u} \cap B} \varphi\left(x, u^{+}, u^{-}, n_{u}\right) \mathrm{d} \mathcal{H}^{1}, \quad u \in B V\left(T^{2}, \mathbb{Z}^{N}\right), B \in \mathcal{B}\left(T^{2}\right),
$$

with $\varphi: T^{2} \times \mathbb{Z}^{N} \times \mathbb{Z}^{N} \times S^{1} \rightarrow[0, \infty)$ defined by

$$
\varphi(x, i, j, n)=\limsup _{\rho \rightarrow 0^{+}} \frac{1}{\rho} \min \left\{F\left(u, \overline{Q_{\rho}^{n}}(x)\right): u \in \mathcal{X}\right\}
$$

and

$$
\mathcal{X}=\left\{u \in B V\left(T^{2}, \mathbb{Z}^{N}\right): u=u_{i j}^{n x} \text { on } T^{2} \backslash Q_{\rho}^{n}(x)\right\} .
$$

To conclude it remains to prove that formula (47) for the density $\varphi$ reduces to 46.

In what follows we will highlight the dependence of the class $\mathcal{X}$ on parameters, setting

$$
\mathcal{X}=\mathcal{X}\left(x, s_{1}, s_{2}, n, \rho\right) \quad \text { with } x \in T^{2}, s_{1}, s_{2} \in \mathbb{Z}^{N}, n \in S^{1}, \rho>0 .
$$

Moreover, when we evaluate $F(u, \cdot)$ on closed sets $\overline{Q_{\rho}^{n}}(x)$ we mean that we define it by outer approximation considering open sets $A \in \mathcal{A}\left(T^{2}\right)$ such that $Q_{\rho}^{n}(x) \subset \subset A$.

The proof of formula (46) is split into three steps.

STEP 1: $\varphi$ does not depend separately on $s_{1}, s_{2} \in \mathbb{Z}^{N}$, but only on $s:=s_{1}-s_{2}$. Fix $(x, n) \in T^{2} \times S^{1}$. For every $s_{1}, s_{2} \in \mathbb{Z}^{N}, A \in \mathcal{A}\left(T^{2}\right)$ such that $Q_{\rho}^{n}(x) \subset \subset A$ and every $u \in \mathcal{X}\left(x, s_{1}, s_{2}, n, \rho\right)$, let $\left\{u_{h}\right\}$ be a recovery sequence for $F(u, A)$. For every $c \in \mathbb{Z}^{N}$ it follows that $u_{h}+c \rightarrow u+c$ in $L^{1}\left(T^{2}\right)$ and

$$
F(u+c, A) \leqslant \lim _{h \rightarrow \infty} F_{\varepsilon_{h}}\left(u_{h}+c, A\right)=\lim _{h \rightarrow \infty} F_{\varepsilon_{h}}\left(u_{h}, A\right)=F(u, A),
$$

where we have used the $\Gamma$-liminf inequality and the integer translation invariance of $F_{\varepsilon_{h}}$. Swapping $u$ and $u+c$ we get the opposite inequality, so that the functional $F$ is also integer translation invariant.

Choose $c=-s_{2}$ and set $v:=u+c$. It follows that $v \in \mathcal{X}\left(x, s_{1}-s_{2}, 0, n, \rho\right)$ and

$$
\min \left\{F\left(u, \overline{Q_{\rho}^{n}}(x)\right): u \in \mathcal{X}\left(x, s_{1}, s_{2}, n, \rho\right)\right\}=\min \left\{F\left(v, \overline{Q_{\rho}^{n}}(x)\right): v \in \mathcal{X}\left(x, s_{1}-s_{2}, 0, n, \rho\right)\right\}
$$

and hence

$$
\varphi\left(x, s_{1}, s_{2}, n\right)=\varphi\left(x, s_{1}-s_{2}, 0, n\right) .
$$

STEP 2: $\varphi$ does not depend on $x \in T^{2}$. Fix $(s, n) \in \mathbb{Z}^{N} \times S^{1}$ and set, with a little abuse of notations, $\mathcal{X}(x, s, n, \rho)=\mathcal{X}(x, s, 0, n, \rho)$ and $\varphi(x, s, n)=\varphi(x, s, 0, n)$. For every $x_{0}, x_{1} \in T^{2}$, 
$u \in \mathcal{X}\left(x_{0}, s, n, \rho\right)$ and $A \in \mathcal{A}\left(T^{2}\right)$ such that $Q_{\rho}^{n}\left(x_{0}\right) \subset \subset A$, we define

$$
\tau[u](x):=u\left(x+x_{0}-x_{1}\right) \quad \text { and } \quad \tau^{-1}(A):=A+\left(x_{1}-x_{0}\right),
$$

so that $\tau[u] \in \mathcal{X}\left(x_{1}, s, n, \rho\right)$ and $Q_{\rho}^{n}\left(x_{1}\right) \subset \subset \tau^{-1}(A) \in \mathcal{A}\left(T^{2}\right)$.

Let $\left\{u_{h}\right\}$ be a recovery sequence for $F(u, A)$. Then $\tau\left[u_{h}\right] \rightarrow \tau[u]$ in $L^{1}\left(T^{2}\right)$ and by a change of variables and the $\Gamma$-liminf inequality for the functional $F_{\varepsilon_{h}}$ we get

$$
F(u, A)=\lim _{h \rightarrow \infty} F_{\varepsilon_{h}}\left(u_{h}, A\right)=\lim _{h \rightarrow \infty} F_{\varepsilon_{h}}\left(\tau\left[u_{h}\right], \tau^{-1}(A)\right) \geqslant F\left(\tau[u], \tau^{-1}(A)\right) .
$$

Similarly, we get the opposite inequality, so that we conclude that

$$
\min \left\{F\left(u, \overline{Q_{\rho}^{n}}\left(x_{0}\right)\right): u \in \mathcal{X}\left(x_{0}, s, n, \rho\right)\right\}=\min \left\{F\left(v, \overline{Q_{\rho}^{n}}\left(x_{1}\right)\right): v \in \mathcal{X}\left(x_{1}, s, n, \rho\right)\right\}
$$

and hence

$$
\varphi\left(x_{0}, s, n\right)=\varphi\left(x_{1}, s, n\right)
$$

STEP 3: $\varphi(s, n)=F\left(u_{s}^{n}, Q^{n}\right)$ for every $s \in \mathbb{Z}^{N}$ and $n \in S^{1}$. This step trivially follows from the integral representation of $F$ and from the fact that $\varphi$ does not depend on $x$. In fact,

$$
F\left(u_{s}^{n}, Q^{n}\right)=\int_{S u_{s}^{n} \cap Q^{n}} \varphi(s, n) \mathrm{d} \mathcal{H}^{1}=\varphi(s, n),
$$

and hence the proof of Theorem 8 is complete.

REMARK 9 Note that by the fact that $F$ is a $\Gamma$-limit we automatically know that it is lower semicontinuous with respect to the $L^{1}$ topology. As a consequence its energy density $\varphi$ defined in (46) must satisfy the necessary and sufficient condition for the lower semicontinuity of functionals defined on partitions, the so called $B V$-ellipticity. We say that $\varphi$ is BV-elliptic if it satisfies the following condition:

$$
\varphi(s, n)=\min \left\{\int_{S_{u} \cap Q^{n}} \varphi\left([u], n_{u}\right) \mathrm{d} \mathcal{H}^{1}: u \in \mathcal{X}\left(Q^{n}\right)\right\}
$$

for all $s \in \mathbb{Z}^{N}$ and $n \in S^{1}$, i.e., the step function $u_{s}^{n}(x)=s \chi_{\{x \cdot n>0\}}$ minimizes the above minimum problem.

It is proved in [6] that a necessary (but in general not sufficient) condition for the BV-ellipticity is the following pair of properties:

(i) (Subadditivity in $s$ ) For any $n \in S^{1}$ and every $s_{1}, s_{2} \in \mathbb{Z}^{N}$,

$$
\varphi\left(s_{1}+s_{2}, n\right) \leqslant \varphi\left(s_{1}, n\right)+\varphi\left(s_{2}, n\right) .
$$

(ii) (Convexity in $n$ ) For every $s \in \mathbb{Z}^{N}$ the positively homogeneous extension of degree 1 of the function $\varphi(s, \cdot): S^{1} \rightarrow \mathbb{R}$ to the whole of $\mathbb{R}^{2}$ is convex. This condition can be equivalently expressed as

$$
\varphi(s, n) \leqslant l_{1} \varphi\left(s, n_{1}\right)+l_{2} \varphi\left(s, n_{2}\right)
$$

for all $n, n_{1}, n_{2} \in S^{1}$ and $l_{1} n_{1}+l_{2} n_{2}=n$. 


\section{Interfacial microstructure for the cubic lattice}

In this section we consider the case of the functional of dislocations for a cubic lattice and we discuss with an example the important difference between the case under consideration and the scalar case presented in Section 2.2, which is due to the vector nature of slip fields and the anisotropy of the elastic interactions of the crystal. More precisely, we find that the structure of the core of a transition between two phases now plays a fundamental role in the optimization of the limit energy, depending on the orientation of the normal to dislocation lines.

We consider a crystal with a cubic lattice, in which the crystallographic slip is driven by two slip directions $e_{1}$ and $e_{2}$ (the canonical basis of $\mathbb{R}^{2}$ ). Then the functional $F_{\varepsilon}$ of dislocations, rescaled by the factor $|\log \varepsilon|$, reduces to

$F_{\varepsilon}(u)=\frac{1}{|\log \varepsilon|} \int_{T^{2}} \int_{T^{2}}(u(x)-u(y))^{T} \mathrm{~J}(x-y)(u(x)-u(y)) \mathrm{d} x \mathrm{~d} y+\frac{1}{\varepsilon|\log \varepsilon|} \int_{T^{2}} \operatorname{dist}^{2}\left(u(x), \mathbb{Z}^{2}\right) \mathrm{d} x$,

where $u: T^{2} \rightarrow \mathbb{R}^{2}$ is a 1 -periodic vector field and the kernel $\mathrm{J}$ is given by

$$
\mathrm{J}(x)=\sum_{k \in \mathbb{Z}^{2}} \mathrm{~J}_{0}(x+k), \quad \mathrm{J}_{0}(x)=\frac{1}{8 \pi(1-v)|x|^{3}}\left(\begin{array}{cc}
v+1-3 v \frac{x_{2}^{2}}{|x|^{2}} & 3 v \frac{x_{1} x_{2}}{|x|^{2}} \\
3 v \frac{x_{1} x_{2}}{|x|^{2}} & v+1-3 v \frac{x_{1}^{2}}{|x|^{2}}
\end{array}\right) .
$$

By Theorem 2 the $\Gamma$-limit functional $F$ of $F_{\varepsilon}$ exists up to subsequences in $\varepsilon$ and can be represented, for every slip field $u \in B V\left(T^{2}, \mathbb{Z}^{2}\right)$, by the integral on the singular set $S_{u}$ of a density function $\varphi(s, n)$, which is implicitly defined, for every $s=\left(s_{1}, s_{2}\right) \in \mathbb{Z}^{2}$ and $n \in S^{1}$, as the value $F\left(u_{s}^{n}, Q^{n}\right)$, where $u_{s}^{n}=s \chi_{\{x \cdot n>0\}}$ is a step field. The characterization of $\varphi$ is equivalent to the construction of a recovery sequence for $F$, i.e., a transition $u_{\varepsilon}$ such that

$$
u_{\varepsilon} \stackrel{\varepsilon \rightarrow 0}{\longrightarrow} u_{s}^{n} \quad \text { and } \quad \lim _{\varepsilon \rightarrow 0} F_{\varepsilon}\left(u_{\varepsilon}, Q^{n}\right)=F\left(u_{s}^{n}, Q^{n}\right)=\varphi(s, n) .
$$

Let us fix $s=(1,1)$. In view of what happens in the scalar case let us try as a possible recovery sequence the mollification at scale $\varepsilon$ of the step function $u_{s}^{n}$. In other words, let us compute the limit energy for a flat transition. We choose an arbitrary function $\phi \in C_{0}^{\infty}\left(Q^{n}\right)$ such that $\phi \geqslant 0$, $\int_{Q^{n}} \phi \mathrm{d} x=1$ and set $u_{\varepsilon}:=u_{s}^{n} * \phi_{\varepsilon}$ with $\phi_{\varepsilon}(x)=\varepsilon^{-2} \phi(x / \varepsilon)$. Since $u_{\varepsilon}$ agrees with $u_{s}^{n}$ outside the $\varepsilon$-neighborhood of $S_{u_{s}^{n}}$, it follows that

$$
\lim _{\varepsilon \rightarrow 0} \frac{1}{\varepsilon|\log \varepsilon|} \int_{Q^{n}} \operatorname{dist}^{2}\left(u_{\varepsilon}, \mathbb{Z}^{2}\right) \mathrm{d} x \leqslant \lim _{\varepsilon \rightarrow 0} \frac{C \varepsilon}{\varepsilon|\log \varepsilon|}=0
$$

and

$$
\begin{aligned}
\lim _{\varepsilon \rightarrow 0} F_{\varepsilon}\left(u_{\varepsilon}, Q^{n}\right) & =\lim _{\varepsilon \rightarrow 0} \frac{1}{|\log \varepsilon|} \int_{Q^{n}} \int_{Q^{n}}\left(u_{\varepsilon}(x)-u_{\varepsilon}(y)\right)^{T} \mathbf{J}_{0}(x-y)\left(u_{\varepsilon}(x)-u_{\varepsilon}(y)\right) \mathrm{d} x \mathrm{~d} y \\
& =s^{T} \gamma(n) s=\gamma_{11}(n)+\gamma_{22}(n)+2 \gamma_{12}(n)=: F_{\text {flat }}\left(u_{s}^{n}\right),
\end{aligned}
$$

where $\gamma(n)$ is the anisotropic line tension matrix defined by

$$
\gamma_{i j}(n):=2 \int_{x \cdot n=1}\left(\mathrm{~J}_{0}(x)\right)_{i j} \mathrm{~d} \mathcal{H}^{1}, \quad i, j=1,2 .
$$


This formula for $\gamma(n)$ can be obtained as in the scalar case (see [14] for details). Roughly speaking, the terms $\gamma_{11}(n)$ and $\gamma_{22}(n)$ in 50 represent the energy cost of a unit jump in the first and in the second component of $u_{s}^{n}$ respectively, and $\gamma_{12}(n)$ is the energy associated to a simultaneous jump of the two components.

The matrix $\gamma(n)$ can be explicitly computed and is given by

$$
\gamma(n)=\gamma(\theta)=\frac{1}{4 \pi(1-v)}\left(\begin{array}{cc}
2-2 \nu \sin ^{2} \theta & v \sin 2 \theta \\
v \sin 2 \theta & 2-2 v \cos ^{2} \theta
\end{array}\right),
$$

where $n \in S^{1}$ is given by $n=(\cos \theta, \sin \theta)$ with $\theta \in[-\pi, \pi)$. Since the Poisson ratio $v$ of the crystal ranges in $(-1,1 / 2)$, it is not difficult to check that the matrix $\gamma(n)$ is positive definite, as it should be, and the entries $\gamma_{11}(\theta)$ and $\gamma_{22}(\theta)$ are both strictly positive for every $\theta \in[-\pi, \pi)$. The relevant fact is that the entry $\gamma_{12}(\theta)$ changes sign. For instance if $v>0$, then $\gamma_{12}(\theta)$ is negative if $\theta<0$. This property has an important consequence. In fact, if $\theta>0$ we can obtain a limit energy smaller than $F_{\text {flat }}\left(u_{s}^{n}\right)$ by approximating $u_{s}^{n}$ with a slip field whose components never jump together. For example, fix $\theta=\pi / 4$ and suppose that $v>0$. For every $\delta \ll 1$ we consider the slip field $u_{\delta}$ in Figure 2. The singular set $S_{u_{s}^{n}}$ is replaced by its $\delta$-neighborhood $N_{\delta}$, in which the third phase

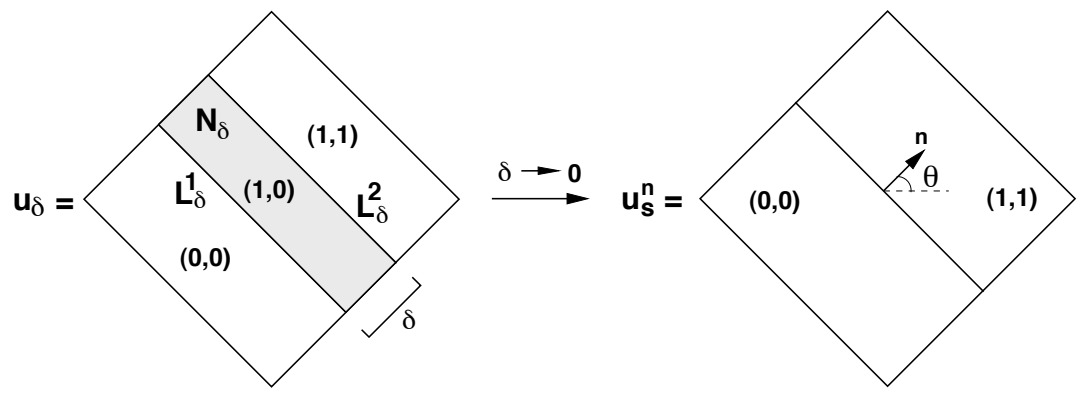

FIG. 2. Splitting the jumps is energetically favorable.

$(1,0)$ appears and the jump sets of the two components of $u_{\delta}$ (segments $L_{\delta}^{1}$ and $L_{\delta}^{2}$ respectively) are disjoint. We choose a sequence $\delta=\delta_{\varepsilon}$ converging to zero such that $\delta_{\varepsilon} \gg \varepsilon$ (precisely such that $\left.\left|\log \delta_{\varepsilon}\right| /|\log \varepsilon| \rightarrow 0\right)$. Clearly $u_{\delta_{\varepsilon}} * \phi_{\varepsilon} \rightarrow u_{s}^{n}$, where $\phi_{\varepsilon}$ is a mollifier, and since $\gamma_{12}(\theta)>0$ it follows that

$$
\lim _{\varepsilon \rightarrow 0} F_{\varepsilon}\left(u_{\delta_{\varepsilon}} * \phi_{\varepsilon}, Q^{n}\right)=\gamma_{11}(\theta)+\gamma_{22}(\theta)<\gamma_{11}(\theta)+\gamma_{22}(\theta)+2 \gamma_{12}(\theta)=\lim _{\varepsilon \rightarrow 0} F_{\varepsilon}\left(u_{s}^{n} * \phi_{\varepsilon}, Q^{n}\right)
$$

On the other hand, if $\theta<0$ as in Figure 3 then $\gamma_{12}(\theta)<0$. In this case it is better not to split the jump and have the two components jumping together, i.e., with the same notation as above we have

$$
\lim _{\varepsilon \rightarrow 0} F_{\varepsilon}\left(u_{s}^{n} * \phi_{\varepsilon}, Q^{n}\right)=\gamma_{11}(\theta)+\gamma_{22}(\theta)+2 \gamma_{12}(\theta)<\gamma_{11}(\theta)+\gamma_{22}(\theta)=\lim _{\varepsilon \rightarrow 0} F_{\varepsilon}\left(u_{\delta_{\varepsilon}} * \phi_{\varepsilon}, Q^{n}\right) .
$$

Here is the main difference with respect to the scalar case: the anisotropy and the additional phase $(1,0)$ (or equivalently $(0,1))$ make a good transition between $(0,0)$ and $(1,1)$ dependent on the orientation of the interface; in some directions it is better to split the jumps of $u_{s}^{n}$, in others it is 


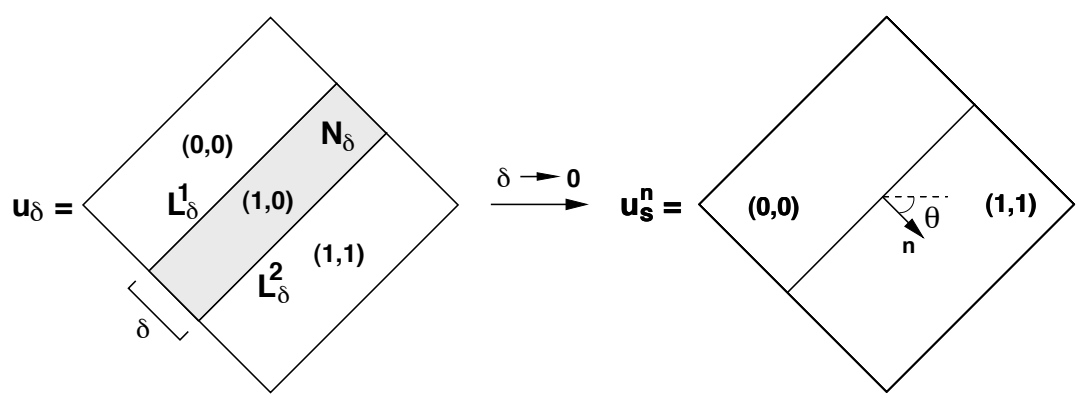

FIG. 3. Piling up the jumps is energetically favorable.

better to pile them up. This suggests that in some cases these two effects may combine and create a more complex good transition.

We now describe an interesting construction, which uses the observations above, for the interface with normal $n=e_{1}$, corresponding to the choice $\theta=0$. Since $\gamma_{12}(0)=0$, in this case the splitting of the jump of $u_{s}^{n}$ has no effect on the limit energy value. We then consider, for every $\sigma \ll 1$, the following three-phase field $u_{\sigma}:=\left(u_{\sigma}^{1}, u_{\sigma}^{2}\right)$, as in Figure 4 The singular set $S_{u_{\sigma}}$ is the union

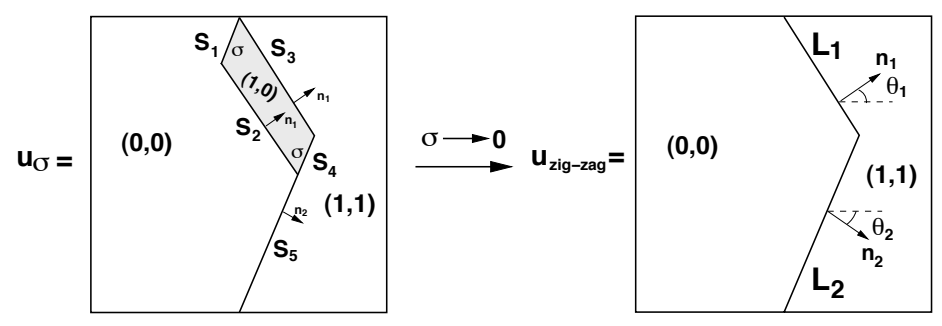

FIG. 4. A new combination of jumps.

of the segments $S_{i}$, with $i=1, \ldots, 5$, the first four of which delimit a small region filled by the third phase $(1,0)$. Note that $u_{\sigma}^{1}$ jumps only across $S_{1}, S_{2}, u_{\sigma}^{2}$ across $S_{3}, S_{4}$, and both across $S_{5}$. Moreover, the normal $n_{1}$ to $S_{2}, S_{3}$, corresponding to $\theta_{1}$, is such that $\gamma_{12}\left(\theta_{1}\right)>0$, the normal $n_{2}$ to $S_{5}$, corresponding to $\theta_{2}$, satisfies $\gamma_{12}\left(\theta_{2}\right)<0$, and the length of $S_{1}$ and $S_{4}$ is of order $\sigma$. We choose a sequence $\sigma=\sigma_{\varepsilon}$ converging to zero such that $\sigma_{\varepsilon} \gg \varepsilon$ and compute the limit energy of a regularization of $u_{\sigma_{\varepsilon}}$. It follows that

$$
\lim _{\varepsilon \rightarrow 0} F_{\varepsilon}\left(u_{\sigma_{\varepsilon}} * \phi_{\varepsilon}, Q^{n}\right)=\left(\gamma_{11}\left(\theta_{1}\right)+\gamma_{22}\left(\theta_{1}\right)\right)\left|L_{1}\right|+\left(\gamma_{11}\left(\theta_{2}\right)+2 \gamma_{12}\left(\theta_{2}\right)+\gamma_{22}\left(\theta_{2}\right)\right)\left|L_{2}\right|,
$$

where $L_{1}, L_{2}$ and $\theta_{1}, \theta_{2}$ denote respectively the jump sets and the angles of the corresponding normals of the phase field $u_{\text {zig-zag }}:=\lim _{\sigma \rightarrow 0} u_{\sigma}$ (see Figure 4). The key point is that we can choose $\theta_{1}$ and $\theta_{2}$ such that

$$
\begin{aligned}
F_{\text {zig-zag }}\left(u_{s}^{n}\right): & =\left(\gamma_{11}\left(\theta_{1}\right)+\gamma_{22}\left(\theta_{1}\right)\right)\left|L_{1}\right|+\left(\gamma_{11}\left(\theta_{2}\right)+2 \gamma_{12}\left(\theta_{2}\right)+\gamma_{22}\left(\theta_{2}\right)\right)\left|L_{2}\right| \\
& <\gamma_{11}(0)+\gamma_{22}(0)=F_{\text {flat }}\left(u_{s}^{n}\right),
\end{aligned}
$$


where the right hand side is the limit energy of a regularization of $u_{s}^{n}$ : the greater length of the jump set $S_{u_{\text {zig-zag }}}$ with respect to $S_{u_{s}^{n}}$ is compensated by the fact that $\gamma_{12}\left(\theta_{2}\right)$ is negative along $L_{2}$, so that the total energy decreases. The existence of such a choice for the angles $\theta_{1}$ and $\theta_{2}$ can be determined analytically or justified as in Remark 10 . This observation finally permits us to construct a good competitor in the approximation of $u_{s}^{n}$.

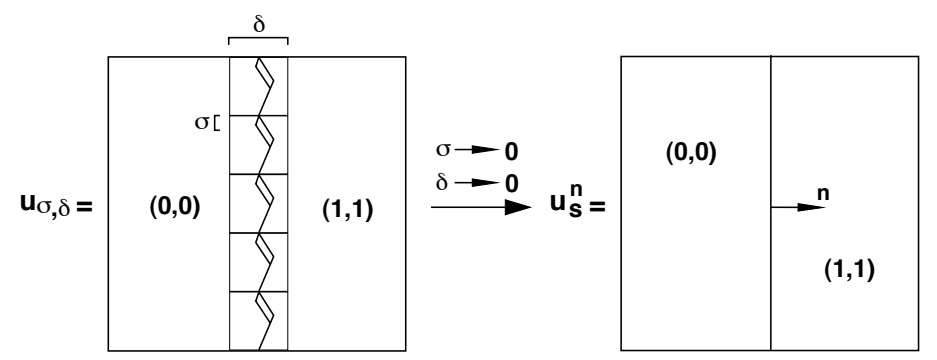

FIG. 5. Zig-zag approximation.

We extend $u_{\sigma}$ onto $\mathbb{R}^{2}$ by periodicity in the variable $x_{2}$ and constant for $\left|x_{1}\right|>1 / 2$, namely equal to $(0,0)$ if $x_{1}<-1 / 2$ and equal to $(1,1)$ if $x_{1}>1 / 2$. For every $\delta$ such that $\sigma \ll \delta \ll 1$, we define the slip field $u_{\sigma, \delta}(x)=u_{\sigma}(x / \delta)$, which is shown in Figure 5. Finally, we choose two sequences $\sigma=\sigma_{\varepsilon}, \delta=\delta_{\varepsilon}$ converging to zero such that $\varepsilon \ll \sigma_{\varepsilon} \ll \delta_{\varepsilon}$, with $\left|\log \sigma_{\varepsilon}\right| /|\log \varepsilon| \rightarrow 0$. By (52) and (53) we then conclude that

$$
\lim _{\varepsilon \rightarrow 0} F_{\varepsilon}\left(u_{\sigma_{\varepsilon}, \delta_{\varepsilon}} * \phi_{\varepsilon}, Q^{n}\right)<\lim _{\varepsilon \rightarrow 0} F_{\varepsilon}\left(u_{s}^{n} * \phi_{\varepsilon}, Q^{n}\right) .
$$

This example shows another important feature of the vector problem: in general the profile of a recovery sequence for the $\Gamma$-limit functional of dislocations cannot be one-dimensional, i.e., it cannot be obtained, as in the scalar case, by means of a simple regularization of the flat interface of step fields, not even in the case of unit jumps.

REMARK 10 The zig-zag construction given above can be motivated by the following argument. We write $\varphi_{\text {split }}(\theta)$ and $\varphi_{\text {pile-up }}(\theta)$ for the two energies obtained by splitting or piling up the jumps as in Figures 2 and 3 respectively, namely

$$
\varphi_{\text {split }}(\theta)=\gamma_{11}(\theta)+\gamma_{22}(\theta), \quad \varphi_{\text {pile-up }}(\theta)=\gamma_{11}(\theta)+\gamma_{22}(\theta)+2 \gamma_{12}(\theta) .
$$

Then the corresponding homogeneous extensions of degree 1 (still denoted by $\varphi_{\text {split }}$ and $\varphi_{\text {pile-up }}$ ) are completely determined by their level 1 set (represented in Figure 6 below). In view of the necessary condition for the BV-ellipticity of energy densities for functionals defined on partitions (see Remark 9) we deduce that the function $\varphi(s, n)$ given by Theorem 2 must be smaller than the

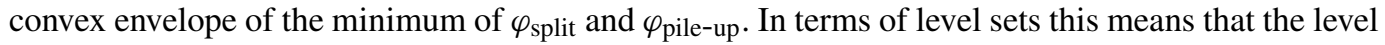
1 set of the homogeneous extension of degree 1 of $\varphi$ must contain the convex envelope of the union

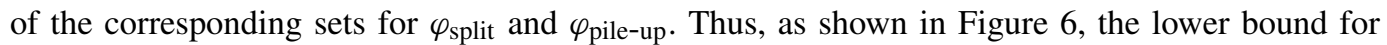
the energy of the step function $u_{s}^{n}$, with $n=e_{1}$ (i.e. $\theta=0$ ) and $s=(1,1)$, as constructed in the zig-zag example above, is nothing other than the approximation of the point in the convex envelope obtained by mixing with inclination $\theta_{1}$ and $\theta_{2}$ the splitting and piling up energy respectively. 


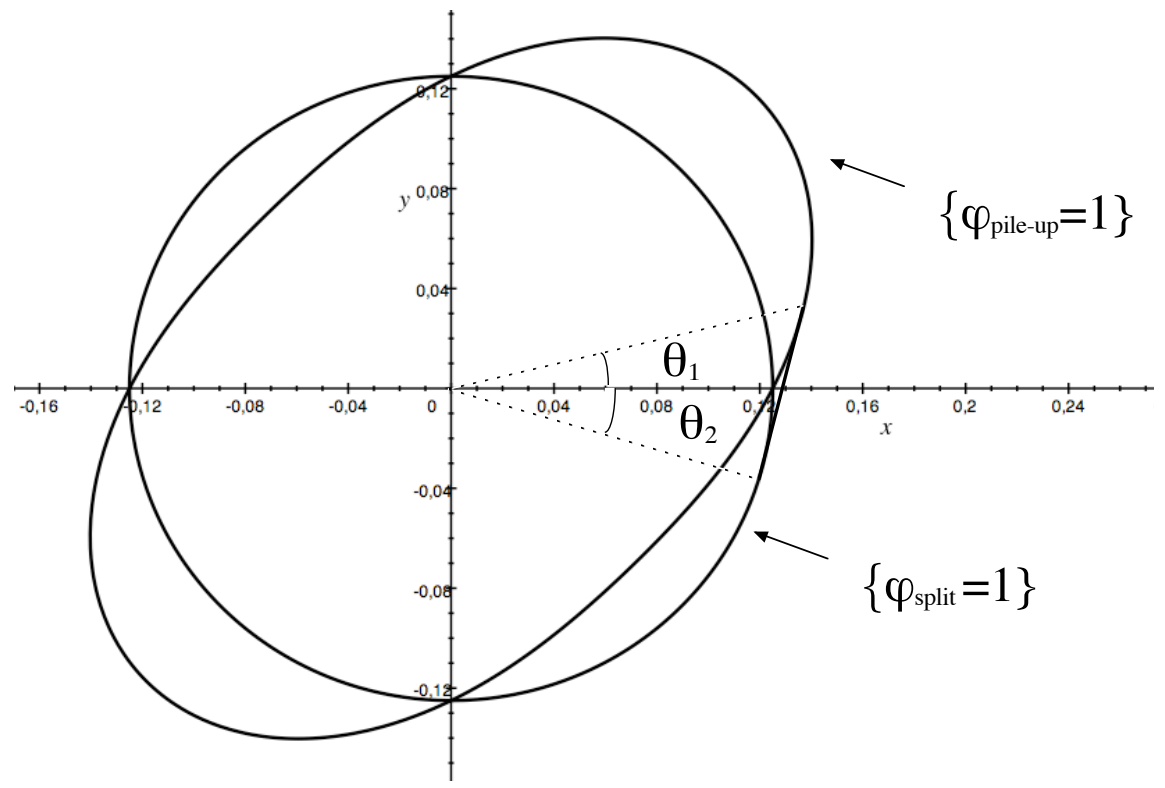

FIG. 6. Zig-zag approximation in terms of the level sets of the energy density.

We conclude this paper by highlighting that a crucial point in the construction above is that for every $u \in B V\left(T^{2}, \mathbb{Z}^{N}\right)$ the energy $F_{\text {flat }}(u)$ obtained by taking the limit of $F_{\varepsilon}\left(u * \phi_{\varepsilon}\right)$ is in general not lower semicontinuous with respect to the $L^{1}$ topology. In other words, the energy density $\varphi_{\text {flat }}(s, n):=s^{T} \gamma(n) s$ of $F_{\text {flat }}$ is not $B V$-elliptic. Clearly its $B V$-elliptic envelope $\bar{\varphi}_{\text {flat }}(s, n)$ is an upper bound for the energy density $\varphi(s, n)$ of the $\Gamma$-limit. In view of the necessary conditions for the $B V$-ellipticity (see Remark 10 ), the convex envelope of the minimum of the homogeneous extensions of degree 1 of $\varphi_{\text {split }}(\theta)$ and $\varphi_{\text {pile-up }}(\theta)$ is a good candidate for $\bar{\varphi}_{\text {flat }}(s, n)$ (or at least is greater than $\bar{\varphi}_{\text {flat }}(s, n)$ and smaller than $\left.\varphi_{\text {flat }}(s, n)\right)$.

The idea is then that if the sequences with low energy are essentially regularizations of some multi-phase field in $B V\left(T^{2}, \mathbb{Z}^{N}\right)$ without too fine microstructures, then one could first reduce the functional $F_{\varepsilon}$ to the sharp interface limit (given by $F_{\text {flat }}$ ) and then relax. This gives rise to the following conjecture.

Conjecture The energy density of the $\Gamma$-limit of $F_{\varepsilon}, \varphi(s, n)$, is given by the $B V$-elliptic envelope of $\varphi_{\text {flat }}(s, n)=s^{T} \gamma(n) s$, i.e.

$$
\varphi(s, n)=\bar{\varphi}_{\text {flat }}(s, n):=\min \left\{\int_{S_{u} \cap Q^{n}} \varphi_{\text {flat }}\left([u], n_{u}\right) \mathrm{d} \mathcal{H}^{1}: u \in \mathcal{X}\left(Q^{n}\right)\right\}
$$

for all $s \in \mathbb{Z}^{N}$ and $n \in S^{1}$, where the notations are those of Remark 9

\section{REFERENCES}

1. Alberti, G., \& Bellettini, G. A non-local anisotropic model for phase transitions: asymptotic behavior of rescaled energies. Eur. J. Appl. Math. 9 (1998), 261-284. Zbl 0932.49018 MR 1634336 
2. Alberti, G., \& Bellettini, G. A non-local anisotropic model for phase transitions. The optimal profile problem. Math. Ann. 310 (1998), 527-560. Zbl 0891.49021 MR 1612250

3. Alberti, G., Bellettini, G., Cassandro, M., \& Presutti, E. Surface tension in Ising systems with Kac potential. J. Statist. Phys. 82 (1996), 743-796. Zbl 1042.82539 MR 1372427

4. Alberti, G., Bouchitté, G., \& Seppecher, P. Phase transitions with line tension effect. Arch. Ration. Mech. Anal. 144 (1998), 1-46. Zbl 0915.76093 MR 1657316

5. Alberti, G., Bouchitté, G., \& Seppecher, P. Un résultat de perturbations singulières avec la norme $H^{1 / 2}$. C. R. Acad. Sci. Paris 319 (1994), 333-338. Zbl 0845.49008 MR 1289307

6. Ambrosio, L., \& Braides, A. Functionals defined on partitions of sets of finite perimeter, I: integral representation and $\Gamma$-convergence. J. Math. Pures Appl. 69 (1990), 285-305. Zbl 0676.49028 MR 1070481

7. Ambrosio, L., \& Braides, A. Functionals defined on partitions of sets of finite perimeter, II: semicontinuity, relaxation and homogenization. J. Math. Pures Appl. 69 (1990), 307-333. Zbl 0676.49029 MR 1070482

8. BACON, D. J., \& Hull, D. Introduction to Dislocations. Int. Ser. Materials Science and Technology 37, Elsevier (1984).

9. BALDO, S. Minimal interface criterion for phase transitions in mixtures of Cahn-Hilliard fluids. Ann. Inst. H. Poincaré Anal. Non Linéaire 7 (1990), 67-90. Zbl 0702.49009 MR 1051228

10. Bouchitté, G. Singular perturbations of variational problems arising from a two-phase transition model. Appl. Math. Optim. 21 (1990), 289-314. Zbl 0695.49003 MR 1036589

11. Braides, A. $\Gamma$-convergence for Beginners. Oxford Lecture Ser. Math. Appl. 22, Oxford Univ. Press (2002). Zbl pre01865939 MR 1968440

12. CAhn, J. W., \& Hilliard, J. E. Free energy of a non-uniform system I-Interfacial free energy. J. Chem. Phys. 28 (1958), 258-267.

13. De Giorgi, E., \& LetTa, G. Une notion générale de convergence faible pour des fonctions croissantes d'ensemble. Ann. Scuola Norm. Sup. Pisa Cl. Sci. 4 (1977), 61-99. Zbl 0405.28008 MR 0466479

14. Garroni, A., \& MÜller, S. $\Gamma$-limit of a phase field model of dislocations. SIAM J. Math. Anal. 36 (2005), 1943-1964. Zbl 1094.82008 MR 2178227

15. Garroni, A., \& MÜller, S. A variational model for dislocations in the line tension limit. Arch. Ration. Mech. Anal. 181 (2006), 535-578. Zbl pre05051266 MR 2231783

16. Koslowski, M., Cuitino, A. M., \& ORtiz, M. A phase-field theory of dislocation dynamics, strain hardening and hysteresis in ductile single crystals. J. Mech. Phys. Solids 50 (2002), 2597-2635. Zbl 1094.74563 MR 1935021

17. Koslowski, M., \& Ortiz, M. A multi-phase field model of planar dislocation networks. Model Simul. Mater. Sci. Engrg. 12 (2004), 1087-1097.

18. KuRZKe, M. A non local singular perturbation problem with periodic well potential. ESAIM Control Optim. Calc. Var. 12 (2006), 52-63. Zbl 1107.49016 MR 2192068

19. ModicA, L. The gradient theory of phase transition and the minimal interface criterion. Arch. Ration. Mech. Anal. 98 (1987), 123-142. Zbl 0616.76004 MR 0866718

20. Modica, L., \& Mortola, S. Un esempio di $\Gamma$-convergenza. Boll. Un. Mat. Ital. 14 (1977), 285-299. Zbl 0356.49008 MR 0445362 